\title{
The Distance between Points of a Solution of a Second Order Linear Differential Equation Satisfying General Boundary Conditions
}

\author{
Pedro Almenar ${ }^{1}$ and Lucas Jódar ${ }^{2}$ \\ ${ }^{1}$ Vodafone Central Network and Operations, P. E. Castellana Norte, 28050 Madrid, Spain \\ ${ }^{2}$ Instituto Universitario de Matemática Multidisciplinar, Universitat Politècnica de València, Camino de Vera S/N, \\ 46022 Valencia, Spain
}

Correspondence should be addressed to Lucas Jódar; ljodar@imm.upv.es

Received 14 December 2013; Accepted 15 April 2014; Published 15 May 2014

Academic Editor: Csaba Varga

Copyright (C) 2014 P. Almenar and L. Jódar. This is an open access article distributed under the Creative Commons Attribution License, which permits unrestricted use, distribution, and reproduction in any medium, provided the original work is properly cited.

This paper presents a method to obtain lower and upper bounds for the minimum distance between points $a$ and $b$ of the solution of the second order linear differential equation $y^{\prime \prime}+q(x) y=0$ satisfying general separated boundary conditions of the type $a_{11} y(a)+a_{12} y^{\prime}(a)=0$ and $a_{21} y(b)+a_{22} y^{\prime}(b)=0$. The method is based on the recursive application of a linear operator to certain functions, a recursive application that makes these bounds converge to the exact distance between $a$ and $b$ as the recursivity index grows. The method covers conjugacy and disfocality as particular cases.

\section{Introduction}

In a recent paper of the authors (see [1]) it was shown that the recursive application of the operator $P: C[a, b] \rightarrow C^{2}[a, b]$ defined by

$$
P f=\int_{a}^{x} \int_{t}^{b} q(s) f(s) d s d t
$$

where $q(x)$ is continuous on $[a, b]$ and strictly positive almost everywhere on that same interval, provided a method to determine if the second order linear differential equation

$$
y^{\prime \prime}+q(x) y=0
$$

was either left disfocal or left nondisfocal in the interval $[a, b]$, the concept of left disfocal alluding to the nonexistence of a nontrivial solution $y(x)$ of $(2)$ with zeroes in $[a, b]$ such that $y^{\prime}(b)=0$ (a similar definition exists for right disfocal). The method was based on two features of the operator $P$, namely,

(i) the fact that $P$ is positive and monotonic, so that for $f>y>g>0$ on $] a, b\left[\right.$ one has $P^{k} f(x)>P^{k} y(x)>$ $P^{k} g(x)$ on $\left.] a, b\right]$; (ii) the fact that $\lim _{k \rightarrow \infty} P^{k} f(b)=\infty$ if (2) is left nondisfocal in an interval interior to $[a, b]$ and $\lim _{k \rightarrow \infty} P^{k} f(x)=0$ for any value of $x \in[a, b]$ as long as (2) is left disfocal in $[a, b]$.

The purpose of this paper is to extend the results of [1] to (2) with the more general boundary conditions:

$$
a_{11} y(a)+a_{12} y^{\prime}(a)=0, \quad a_{21} y(b)+a_{22} y^{\prime}(b)=0,
$$

which contain conjugacy $(y(a)=y(b)=0)$ and nondisfocality as particular cases, by means of the recursive application of the operator $L: C[a, b] \rightarrow C^{2}[a, b]$ defined by

$$
L f=\int_{a}^{b} G(x, t) q(t) f(t) d t,
$$

where $G(x, t)$ (or $G_{a b}(x, t)$, as we will name it in some sections) is the Green function of the problem

$$
\begin{gathered}
y^{\prime \prime}=-g(x), \quad a_{11} y(a)+a_{12} y^{\prime}(a)=0, \\
a_{21} y(b)+a_{22} y^{\prime}(b)=0 .
\end{gathered}
$$


In consequence this paper will yield criteria (in fact infinitely many) to obtain lower and upper bounds for the minimum distance between points $a$ and $b$ satisfying (3) when $y(x)$ is a solution of (2), bounds of which will be shown to converge to the exact values of such a minimum distance as the index $k$ grows. Besides extending the results of [1] to the problem (2)-(3), other results based on the same strategies will also be introduced and it will be shown that they improve the results of [1] in many cases.

Operators of the type (4) have often been used in the determination of Lyapunov inequalities for different types of equations and boundary conditions since Nehari [2] first noted that a solution $y$ of (2) such that $y(a)=y(b)=0$ satisfied

$$
y(x)=\int_{a}^{b} G(x, t) q(t) y(t) d t,
$$

with

$$
G(x, t)=\frac{\min (x-a, t-a) \min (b-x, b-t)}{b-a},
$$

which implied

$$
\begin{aligned}
|y(x)| & \leq \int_{a}^{b}|G(x, t)| q(t)|y(t)| d t \\
& <\|y\|_{\infty} \int_{a}^{b}|G(t, t)| q(t) d t ;
\end{aligned}
$$

that is,

$$
1<\int_{a}^{b}|G(t, t)| q(t) d t .
$$

Beesack [3], Hinton [4], Levin [5, 6], and Reid [7-9] are other remarkable examples of such a use, as the excellent monography on Lyapunov inequalities of [10, Chapter 1] shows. Although formulae like (6) can be applied recursively if $q(x) \geq 0$ and $G(x, t) \geq 0$ to obtain more complex versions of (9), the fact is that the iterative application of $L$ has rarely been proposed in any papers, with the exception of Harris [11], who suggested its application for the disfocal case $-y(a)=$ $y^{\prime}(b)=0$ or $y^{\prime}(a)=y(b)=0$-without getting to prove that it guaranteed any improvement. We will show in this paper that under certain conditions ( 3 ) the function $G(x, t)$ is positive and the recursive application is possible and provides lower and upper bounds which improve all existing results as of today. We will also show that even in the case that $G(x, t)$ gets to be negative it is still sometimes possible to obtain upper and lower bounds for the distance between $a$ and $b$, which are as close to the real distance between $a$ and $b$ as desired.

It is also worth remarking the significant interest that the calculation of lower bounds (i.e., Lyapunov kind-of inequalities) has enjoyed in comparison with the problem of the determination of upper bounds, regardless of the type of boundary conditions (3). This fact was already noted by Došlý in [12] for the conjugate case $(y(a)=y(b)=0)$ and by the authors in $[1,13,14]$ for the nondisfocal case. References $[12,15-20]$ are notable exceptions to this trend.
As indicated in the first paragraph, throughout the paper we will assume that $q(x)$ is continuous on an interval $I \subset R$ such that $[a, b] \subset I$ and that $q(x)$ is strictly positive almost everywhere on $[a, b]$. This allows defining the internal product

$$
\langle f, g\rangle=\int_{a}^{b} q(x) f(x) g(x) d x,
$$

for $f, g$ continuous on $[a, b]$ (it is easy to prove that (10) satisfies all the conditions required by an internal product) and the associated norm $\|\cdot\|_{2}$ defined by

$$
\|f\|_{2}=\left(\int_{a}^{b} q(x) f^{2}(x) d x\right)^{1 / 2} .
$$

Likewise, we will use the notation $L$ to name the operator defined in (4), $L f$ or $L\{f\}$ to name the function with domain $[a, b]$ resulting from the application of $L$ to $f(x) \in C[a, b]$, and $L f(x)$ or $L\{f\}(x)$ to name the value of the function $L f$ at the point $x$ and $L_{\alpha, \beta}$ when other extremes of integration $\alpha$, $\beta$ potentially different from $a, b$ are used in (4). In that latter case, any $\|\cdot\|_{2}$ norm appearing in the same formula will be assumed to be calculated with $\alpha$ and $\beta$ as integration extremes in (11).

We will say that the points $a^{\prime}, b^{\prime}$ are interior to an interval $[a, b]$ if $a^{\prime}, b^{\prime} \in[a, b]$ and $\left[a^{\prime}, b^{\prime}\right] \neq[a, b]$.

The organization of the paper is as follows. Section 2 will present the main properties of the operator $L$. Sections 3 and 4 will apply these properties in different ways to find upper and lower bounds for the minimum distance between points $a$ and $b$ satisfying (3). Section 5 will introduce some formulae which simplify the calculations required in Section 3. Section 6 will apply the method to several examples. Finally Section 7 will draw several conclusions.

\section{The Operator $L f=\int_{a}^{b} G(x, t) q(t) f(t) d t$}

The purpose of this section will be to present the main properties of the operator $L$ defined in (4) for $q(x)$ as specified in the Introduction. As was done in [1], for the sake of clarity such properties will be presented in several lemmas which will lead to Theorem 5, which can be regarded as the key result of this section.

Lemma 1. The operator $L f$ is linear. In addition, if $G(x, t)$ is positive almost everywhere for $t \in[a, b]$, then $L$ is positive and monotonic.

Proof. The linearity of $L$ is quite evident. If $G(x, t)$ is positive almost everywhere for $t \in[a, b]$, the positiveness (and in consequence monotonicity) of $L$ is a consequence of $q(x)$ being positive almost everywhere on $[a, b]$ by hypothesis.

Lemma 2. The operator $L f$ is compact.

Proof. From [21, Theorem 7.2.6] and the fact that $G(x, t)$ is continuous on $[a, b] \times[a, b]$ it is straightforward to show that $L$ is compact with the $\|\cdot\|_{\infty}$ norm and therefore with the $\|\cdot\|_{2}$ norm defined in (11). 
Lemma 3. If any of the following conditions

$$
\begin{gathered}
a_{11}=0, \quad a_{21}=0, \\
a_{11}=0, \quad \frac{a_{22}}{a_{21}}(b-a)+1 \neq 0, \\
\frac{-a_{12}}{a_{11}}(b-a)+1 \neq 0, \quad a_{21}=0, \\
\frac{a_{12}}{a_{11}}\left(\frac{a_{22}}{a_{21}}(b-a)+1\right)-\frac{a_{22}}{a_{21}} \neq 0
\end{gathered}
$$

are met, then the operator $L f$ is self-adjoint.

Proof. Let us define $y_{1}(x)$ as the solution of

$$
y^{\prime \prime}=0, \quad a_{11} y(a)+a_{12} y^{\prime}(a)=0,
$$

and $y_{2}(x)$ as the solution of

$$
y^{\prime \prime}=0, \quad a_{21} y(b)+a_{22} y^{\prime}(b)=0 .
$$

A straightforward calculation shows that if any of the conditions (12) are met, then the Wronskian of $y_{1}$ and $y_{2}$, namely,

$$
W=y_{1}(x) y_{2}^{\prime}(x)-y_{1}^{\prime}(x) y_{2}(x),
$$

is not zero, which implies that $y_{1}$ and $y_{2}$ are not linearly dependent and therefore no nontrivial solutions of (2)-(3) exist. In consequence from [22, Theorem IX.3] one has

$$
G(x, t)= \begin{cases}\frac{-y_{1}(x) y_{2}(t)}{y_{1}(a) y_{2}^{\prime}(a)-y_{1}^{\prime}(a) y_{2}(a)}, & x<t, \\ \frac{-y_{1}(t) y_{2}(x)}{y_{1}(a) y_{2}^{\prime}(a)-y_{1}^{\prime}(a) y_{2}(a)}, & x \geq t .\end{cases}
$$

Now, in order to prove self-adjointness, we need to prove that, given $f, g \in C[a, b],\langle L f, g\rangle=\langle f, L g\rangle$. Thus, from (10) we have

$$
\begin{aligned}
\langle L f, g\rangle & =\int_{a}^{b} q(x) L f(x) g(x) d x \\
& =\int_{a}^{b} q(x) g(x) \int_{a}^{b} G(x, t) q(t) f(t) d t d x .
\end{aligned}
$$

Combining (17) and (16) one gets to

$$
\begin{aligned}
\langle L f, g\rangle= & \frac{-1}{y_{1}(a) y_{2}^{\prime}(a)-y_{1}^{\prime}(a) y_{2}(a)} \\
& \times \int_{a}^{b} q(x) g(x) y_{2}(x) \int_{a}^{x} q(t) y_{1}(t) f(t) d t d x \\
& +\frac{-1}{y_{1}(a) y_{2}^{\prime}(a)-y_{1}^{\prime}(a) y_{2}(a)} \\
& \times \int_{a}^{b} q(x) g(x) y_{1}(x) \int_{x}^{b} q(t) y_{2}(t) f(t) d t d x .
\end{aligned}
$$

And integrating by parts the right-hand side of (18) one finally has

$$
\begin{aligned}
\langle L f, g\rangle= & \frac{-1}{y_{1}(a) y_{2}^{\prime}(a)-y_{1}^{\prime}(a) y_{2}(a)} \\
& \times \int_{a}^{b} \int_{x}^{b} q(t) g(t) y_{2}(t) d t q(x) y_{1}(x) f(x) d x \\
& +\frac{-1}{y_{1}(a) y_{2}^{\prime}(a)-y_{1}^{\prime}(a) y_{2}(a)} \\
& \times \int_{a}^{b} \int_{a}^{x} q(t) g(t) y_{1}(t) d t q(x) y_{2}(x) f(x) d x \\
= & \iint_{a}^{b} q(t) g(t) G(x, t) d t q(x) f(x) d x \\
= & \langle f, L g\rangle .
\end{aligned}
$$

Lemma 4. The operator $L f$ is bounded with the $\|\cdot\|_{\infty}$ norm and verifies

$$
\begin{array}{r}
L f(x) \leq \max \{G(x, t), t \in[a, b]\}\|f\|_{\infty} \int_{a}^{b} q(t) d t, \\
x \in[a, b],
\end{array}
$$

$$
\begin{array}{r}
L f(x) \leq \max \{G(x, t), t \in[a, b]\}\|f\|_{2} \sqrt{\int_{a}^{b} q(t) d t}, \\
x \in[a, b],
\end{array}
$$

where the $\|\cdot\|_{2}$ norm is defined as in (11).

Proof. Equation (20) is an obvious consequence of (4), and (21) follows from (4) by application of Cauchy-Schwarz inequality.

Theorem 5. If any of conditions (12) are met, then the operator Lf has a countably infinite number of eigenvalues $1 / \lambda_{n}$ and associated orthonormal eigenfunctions $\Phi_{n}(x)$, which allow expressing $L^{k} f, k \geq 1$, as

$$
L^{k} f=\sum_{n=1}^{\infty} \frac{\left\langle f, \Phi_{n}\right\rangle}{\lambda_{n}^{k}} \Phi_{n} .
$$

Moreover,

(i) if no nontrivial solution of (2) satisfies (3) either at a, $b$ or at any $a^{\prime}, b^{\prime}$ interior to $[a, b]$, then

$$
\begin{gathered}
\left\|L^{k} f\right\|_{2}^{2}<\|f\|_{2}^{2}, \quad k \geq 1 \\
\lim _{k \rightarrow \infty}\left\|L^{k} f\right\|_{2}=0, \quad \lim _{k \rightarrow \infty} L^{k} f(x)=0,
\end{gathered}
$$


(ii) if there is a nontrivial solution of (2) that satisfies (3) at some $a^{\prime}, b^{\prime}$ interior to $[a, b]$, then

$$
\left\|L^{k} f\right\|_{2}^{2}>\left\langle f, \Phi_{1}\right\rangle^{2}+\sum_{n=2}^{\infty} \frac{\left\langle f, \Phi_{n}\right\rangle^{2}}{\lambda_{n}^{2 k}}, \quad k \geq 1 .
$$

In addition, if $\left\langle f, \Phi_{1}\right\rangle \neq 0$ and $c \in[a, b]$ is such that $\Phi_{1}(c) \neq 0$, then

$$
\begin{aligned}
& \lim _{k \rightarrow \infty}\left\|L^{k} f\right\|_{2}=\infty, \\
& \lim _{k \rightarrow \infty} L^{k} f(c)= \pm \infty,
\end{aligned}
$$

the sign corresponding to that of $\left\langle f, \Phi_{1}\right\rangle \Phi_{1}(c)$.

Proof. Let us consider the eigenvalue problem

$$
\begin{gathered}
\left.y^{\prime \prime}+\lambda q(x) y=0, \quad x \in\right] a, b[ \\
a_{11} y(a)+a_{12} y^{\prime}(a)=0, \quad a_{21} y(b)+a_{22} y^{\prime}(b)=0 .
\end{gathered}
$$

From the theory of ordinary differential equations (see [22, Theorems V.8 and V.9]) it is known that there exist a countably infinite number of eigenvalues $\lambda_{n}$ which form an increasing sequence with $\lim _{n \rightarrow \infty} \lambda_{n}=\infty$, each of which has its corresponding orthonormal (with the norm (11)) eigenfunction $\Phi_{n}(x)$, and that the set of eigenfunctions $\Phi_{n}$ forms an orthonormal basis of $C[a, b]$. Applying the operator $L$ to these eigenfunctions $\Phi_{n}(x)$ and integrating by parts it is easy to show that

$$
L \Phi_{n}=\frac{\Phi_{n}}{\lambda_{n}}, \quad n \geq 1,
$$

which implies that $\Phi_{n}$ are also the eigenfunctions of the operator $L$ with corresponding eigenvalues $1 / \lambda_{n}$. Since from Lemmas 1,2 and $3 L$ is linear, compact, and self-adjoint, we can apply [21, Theorem 7.5.2] and represent $L$ in the canonical form

$$
L f=\sum_{n=1}^{\infty} \frac{\left\langle f, \Phi_{n}\right\rangle}{\lambda_{n}} \Phi_{n} .
$$

Applying $L$ again to (30) it yields

$$
\begin{aligned}
L^{2} f & =\sum_{n=1}^{\infty} \frac{\left\langle L f, \Phi_{n}\right\rangle}{\lambda_{n}} \Phi_{n} \\
& =\sum_{n=1}^{\infty} \frac{\left\langle\sum_{j=1}^{\infty}\left(\left\langle f, \Phi_{j}\right\rangle / \lambda_{j}\right) \Phi_{j}, \Phi_{n}\right\rangle}{\lambda_{n}} \Phi_{n} \\
& =\sum_{n=1}^{\infty} \frac{\left\langle f, \Phi_{n}\right\rangle}{\lambda_{n}^{2}} \Phi_{n},
\end{aligned}
$$

given that $\left\langle\Phi_{i}, \Phi_{i}\right\rangle=1$ and $\left\langle\Phi_{i}, \Phi_{j}\right\rangle=0$ for $i \neq j$. Applying $L$ recursively to (31) one gets to

$$
\begin{aligned}
L^{k} f & =\sum_{n=1}^{\infty} \frac{\left\langle L^{k-1} f, \Phi_{n}\right\rangle}{\lambda_{n}} \Phi_{n} \\
& =\sum_{n=1}^{\infty} \frac{\left\langle\sum_{j=1}^{\infty}\left(\left\langle f, \Phi_{j}\right\rangle / \lambda_{j}^{k-1}\right) \Phi_{j}, \Phi_{n}\right\rangle}{\lambda_{n}} \Phi_{n} \\
& =\sum_{n=1}^{\infty} \frac{\left\langle f, \Phi_{n}\right\rangle}{\lambda_{n}^{k}} \Phi_{n},
\end{aligned}
$$

which is in fact (22). And the application of Parseval's identity (see [21, Lemmas 1.5.14]) to (32) leads to

$$
\left\|L^{k} f\right\|_{2}=\sqrt{\sum_{n=1}^{\infty} \frac{\left|\left\langle f, \Phi_{n}\right\rangle\right|^{2}}{\lambda_{n}^{2 k}}} .
$$

Since $\left\{\lambda_{n}\right\}$ form an increasing sequence, from (33) it is clear that

$$
\left\|L^{k} f\right\|_{2} \leq \sqrt{\sum_{n=1}^{\infty} \frac{\left|\left\langle f, \Phi_{n}\right\rangle\right|^{2}}{\lambda_{1}^{2 k}}}=\frac{\|f\|_{2}}{\lambda_{1}^{k}} .
$$

Now, let us note that if (2) does not have any nontrivial solution that satisfies (3) either at $a, b$ or at any $a^{\prime}, b^{\prime}$ interior to $[a, b]$, the first eigenvalue $\lambda_{1}$ (and therefore all the others) must be strictly greater than 1 . In that case, from (34) one has (23) and

$$
\lim _{k \rightarrow \infty}\left\|L^{k} f\right\|_{2}=0
$$

From (35) and Lemma 4 one gets (24).

Likewise, if there is a nontrivial solution of (2) that satisfies (3) at some $a^{\prime}, b^{\prime}$ interior to $[a, b]$, then $\lambda_{1}$ must be such that $\lambda_{1}<1$. From this and (33) one gets (25). If, in addition, $\left\langle f, \Phi_{1}\right\rangle \neq 0$, from (33) we get to

$$
\lim _{k \rightarrow \infty}\left\|L^{k} f\right\|_{2}=\infty
$$

which is (26). On the other hand, we can write

$$
L^{k} f-\frac{\left\langle f, \Phi_{1}\right\rangle}{\lambda_{1}^{k}} \Phi_{1}=\sum_{n=2}^{\infty} \frac{\left\langle f, \Phi_{n}\right\rangle}{\lambda_{n}^{k}} \Phi_{n} .
$$

We can divide both sides of (37) by $\left\langle f, \Phi_{1}\right\rangle / \lambda_{1}^{k}$ to yield

$$
\begin{aligned}
& \frac{L^{k} f-\left(\left\langle f, \Phi_{1}\right\rangle / \lambda_{1}^{k}\right) \Phi_{1}}{\left\langle f, \Phi_{1}\right\rangle / \lambda_{1}^{k}} \\
& \quad=\frac{\sum_{n=2}^{\infty}\left(\left\langle f, \Phi_{n}\right\rangle / \lambda_{n}^{k}\right) \Phi_{n}}{\left\langle f, \Phi_{1}\right\rangle / \lambda_{1}^{k}} .
\end{aligned}
$$


Applying Parseval's identity to (38) one gets

$$
\begin{aligned}
\left\|\frac{L^{k} f-}{\left.\left.\left\langle f, \Phi_{1}\right\rangle / \Phi_{1}^{k}\right\rangle / \lambda_{1}^{k}\right) \Phi_{1}}\right\|_{2} \\
\quad=\sqrt{\frac{\sum_{n=2}^{\infty}\left(\left|\left\langle f, \Phi_{n}\right\rangle\right|^{2} / \lambda_{n}^{2 k}\right)}{\left|\left\langle f, \Phi_{1}\right\rangle\right|^{2} / \lambda_{1}^{2 k}}} \\
\leq \frac{\|f\|_{2}}{\left|\left\langle f, \Phi_{1}\right\rangle\right|} \frac{\lambda_{1}^{k}}{\lambda_{2}^{k}} .
\end{aligned}
$$

Since $\lambda_{1}<\lambda_{2}$, from (39) one yields

$$
\lim _{k \rightarrow \infty}\left\|\frac{L^{k} f-\left(\left\langle f, \Phi_{1}\right\rangle / \lambda_{1}^{k}\right) \Phi_{1}}{\left\langle f, \Phi_{1}\right\rangle / \lambda_{1}^{k}}\right\|_{2}=0,
$$

which implies that there exists an index $k_{0}$ such that

$$
\left\|\frac{L^{k} f-\left(\left\langle f, \Phi_{1}\right\rangle / \lambda_{1}^{k}\right) \Phi_{1}}{\left\langle f, \Phi_{1}\right\rangle / \lambda_{1}^{k}}\right\|_{2}<\epsilon, \quad k>k_{0} .
$$

From Lemma 4 and (41) one has

$$
\begin{aligned}
& \left|\frac{L^{k} f(c)-\left(\left\langle f, \Phi_{1}\right\rangle / \lambda_{1}^{k}\right) \Phi_{1}(c)}{\left(\left\langle f, \Phi_{1}\right\rangle / \lambda_{1}^{k}\right)}\right| \\
& \quad<\epsilon \max \{G(c, t), t \in[a, b]\} \sqrt{\int_{a}^{b} q(x) d x},
\end{aligned}
$$

for $k>k_{0}+1$; that is,

$$
\begin{aligned}
& \frac{\left|\left\langle f, \Phi_{1}\right\rangle\right|}{\lambda_{1}^{k}} \\
& \quad \times\left(\operatorname{sign}\left(\left\langle f, \Phi_{1}\right\rangle\right) \Phi_{1}(c)-\epsilon \max \{G(c, t), t \in[a, b]\}\right. \\
& \left.\times \sqrt{\int_{a}^{b} q(x) d x}\right) \\
& <L^{k} f(c)<\frac{\left|\left\langle f, \Phi_{1}\right\rangle\right|}{\lambda_{1}^{k}} \\
& \times\left(\operatorname{sign}\left(\left\langle f, \Phi_{1}\right\rangle\right) \Phi_{1}(c)+\epsilon \max \{G(c, t), t \in[a, b]\}\right. \\
& \left.\quad \times \sqrt{\int_{a}^{b} q(x) d x}\right),
\end{aligned}
$$

for $k>k_{0}+1$. Since $\Phi_{1}(c) \neq 0$ by hypothesis and $\lambda_{1}<1,(43)$ leads to (27).
Remark 6. Theorem 5 provides two types of methods to obtain upper and lower bounds for the minimum distance between points $a, b$ satisfying (3), one based on comparing the norm of $L^{k} f$ with some other constants and another based on comparing the value of $L^{k} f$ at concrete points of $[a, b]$ for different functions $f$. These will be addressed separately in the next two sections.

\section{Bounds for the Distance between $a$ and $b$ Based on $\left\|L^{k} f\right\|_{2}$}

As stated before, Theorem 5 provides methods to get upper and lower bounds for the minimum distance between points $a, b$ satisfying (3), which are based on the comparison of $\left\|L^{k} f\right\|_{2}$ with some thresholds for different values of the extremes of integration $a$ and $b$ of (4) and (11).

Thus, on the one hand, if $a, b$ are such that there is a nontrivial solution of (2) which satisfies (3) at some $a^{\prime}, b^{\prime}$ interior to $[a, b]$, from (26) (and as long as $\left\langle f, \Phi_{1}\right\rangle \neq 0$, i.e., $f$ is "close" to $\left.\Phi_{1}\right)$ it is clear that $\left\|L^{k} f\right\|_{2}$ will grow with the index $k$ regardless of the choice of $f$ and accordingly there will be an index $k_{0}$ such that (23) is violated. This allows us to define an algorithm to find progressively better "outer" bounds of the values satisfying (3) by fixing one of them, say $a$, and calculating the minimum values of the extremes $b_{k}$ which give

$$
\left\|L_{a, b_{k}}^{k} f\right\|_{2}=\|f\|_{2},
$$

for different values of $k$, as the following theorem shows.

Theorem 7. Assume that there exists a nontrivial solution $y(x)$ of (2) satisfying (3) at a, $b$. Let the sequence $\left\{b_{k}\right\}$ be defined by (44), where $f$ is a continuous function on $\left[a, b_{k}\right]$ such that $\left\langle f, \Phi_{1}\right\rangle \neq 0$ and $a, b^{\prime}$ fulfill any of conditions (12) for each $b^{\prime} \in$ $\left[b, b_{k}\right]$. Then $b_{k} \geq b$ and $\left\{b_{k}\right\}$ tends to $b$ as $k \rightarrow \infty$.

Proof. The fact that $b_{k} \geq b$ is obvious from (23). Now, let us pick a $\delta>0$. From (26) $\lim _{k \rightarrow \infty}\left\|L_{a, b+\delta}^{k} f\right\|_{2}=\infty$, which means that there exists an index $k_{1}$ such that

$$
\left\|L_{a, b+\delta}^{k} f\right\|_{2}-\|f\|_{2}>0, \quad k>k_{1} .
$$

Given that $\left\|L_{a, b-\delta}^{k} f\right\|_{2}-\|f\|_{2}<0$ for $k \geq 1$, from (23) and the continuity of $\left\|L_{a, x}^{k} f\right\|_{2}-\|f\|_{2}$ on $x$ (continuity guaranteed by the hypothesis), there must exist a value $b_{k} \in[b, b+\delta[$ such that $\left\|L_{a, b_{k}}^{k} f\right\|_{2}-\|f\|_{2}=0$ for each $k>k_{1}$. This proves the second assertion of the theorem.

The application of the method based on Theorem 7 is quite straightforward given that the right-hand side of (44), that is, $\|f\|_{2}^{2}$, is easy to calculate once fixed $f$. However, it is worth remarking that, from (34), the closer the selected function $f$ is to $\Phi_{1}$, the smaller the terms $\left\langle f, \Phi_{n}\right\rangle$ for $n>1$ will be and the faster the sequence $b_{k}$ will converge to $b$. Therefore, although the method can work with any continuous function $f$ such that $\left\langle f, \Phi_{1}\right\rangle \neq 0$, it is desirable to select one that may be as close as possible to the expected $\Phi_{1}$ associated to the problem (28). 
On the other hand, if $a, b$ are such that no nontrivial solution of (2) satisfies (3) either at $a, b$ or at some $a^{\prime}, b^{\prime}$ interior to $[a, b]$, from (24) it is clear that $\left\|L^{k} f\right\|_{2}$ will shrink with the index $k$ regardless of the choice of $f$ and accordingly there will be an index $k_{0}$ such that (25) is violated. As happened before, this allows us to define an algorithm to find progressively better "inner" bounds of the values satisfying (3) by fixing one of them, say $a$, and calculating the values of the extremes $b_{k}$ which give

$$
\left\|L_{a, b_{k}}^{k} f\right\|_{2}=B_{a, b_{k}}, \quad k \geq 1,
$$

where $B_{a, b_{k}}$ is any lower bound of the right-hand side of (25), which has (in turn) a positive lower bound that does not depend on $k$. This is shown in the following theorem.

Theorem 8. Assume that there exists a nontrivial solution $y(x)$ of (2) satisfying (3) at $a, b$. Let the sequence $\left\{b_{k}\right\}$ be defined by (46), where $f$ is a continuous function on $\left[a, b_{k}\right]$ and $a, b^{\prime}$ fulfill any of conditions (12) for each $b^{\prime} \in\left[b_{k}, b\right]$. Then $b_{k} \leq b$ and $\left\{b_{k}\right\}$ tends to $b$ as $k \rightarrow \infty$.

Proof. Again, the fact that $b_{k} \leq b$ is obvious from (25) and (46). Now, let us pick a $\delta>0$. From (24), $\lim _{k \rightarrow \infty}\left\|L_{a, b-\delta}^{k} f\right\|_{2}=0$. This and the fact that $B_{a, b_{k}}$ is bounded below by a positive amount which does not vary with $k$ imply that there exists an index $k_{2}$ such that

$$
\left\|L_{a, b-\delta}^{k} f\right\|_{2}-B_{a, b-\delta}<0, \quad k>k_{2} .
$$

Given that $\left\|L_{a, b+\delta}^{k} f\right\|_{2}-B_{a, b+\delta}>0$ for $k \geq 1$, from (25) and the continuity of $\left\|L_{a, x}^{k} f\right\|_{2}-B_{a, x}$ on $x$, there must exist a value $\left.\left.b_{k} \in\right] b-\delta, b\right]$ such that $\left\|L_{a, b_{k}}^{k} f\right\|_{2}-B_{a, b_{k}}=0$ for each $k>k_{2}$. This proves the second assertion of the theorem.

Unlike what happens with the method based on Theorem 7, the method based on Theorem 8 presents some difficulties in its application due to the fact that neither $\left\langle f, \Phi_{n}\right\rangle$ nor the eigenvalues $\lambda_{n}$ are known. We can overcome them partially by discarding, in the right-hand side of (25), the term of the series of the eigenfunctions beyond the first one, that is, by converting (25) into

$$
\left\|L^{k} f\right\|_{2}^{2} \geq\left\langle f, \Phi_{1}\right\rangle^{2}, \quad k \geq 1
$$

given that the discarded terms are positive (in fact $\left\langle f, \Phi_{1}\right\rangle^{2}$ may be a possible positive lower bound for some types of terms $B_{a, b_{k}}$ used in (46), which does not depend on $k$ ). The resulting method to obtain lower bounds for the distance between $a$ and $b$ will work in the same way as the one described in the previous paragraphs, at the expense of requiring greater values of the index $k$ to violate (48). But even with such a simplification there is still a need to obtain a lower bound for $\left\langle f, \Phi_{1}\right\rangle$ which is not evident at all.

The following lemmas aim at finding cases (fundamentally choices of $f$ and boundary conditions (3)) where the values of $\left\langle f, \Phi_{1}\right\rangle,\left\langle f, \Phi_{n}\right\rangle$, and $\lambda_{n}$ are bounded in a way that allows getting a lower bound for the right-hand side of (25) (or for the right-hand side of (48) if there is no way to do it with (25)), overcoming the problem.
Lemma 9. Let $\lambda_{n}$ and $\Phi_{n}$ be defined as in Theorem 5. Assume that $a, b$ satisfy any of conditions (12) of Lemma 3. Fixed $x \in$ $[a, b]$ and choosing $f(t)=G(x, t)$ and $g(t)=(\delta G(x, t) / \delta x)$ one has

$$
\begin{array}{ll}
\left\langle f, \Phi_{n}\right\rangle=\frac{\Phi_{n}(x)}{\lambda_{n}}, \quad n \geq 1, \\
\left\langle g, \Phi_{n}\right\rangle=\frac{\Phi_{n}^{\prime}(x)}{\lambda_{n}}, \quad n \geq 1 .
\end{array}
$$

Proof. From (10) one has

$$
\left\langle f, \Phi_{n}\right\rangle=\int_{a}^{b} q(t) G(x, t) \Phi_{n}(t) d t, \quad n \geq 1 .
$$

And since $\Phi_{n}$ satisfies (28) one yields

$$
\left\langle f, \Phi_{n}\right\rangle=-\int_{a}^{b} G(x, t) \frac{\Phi_{n}^{\prime \prime}(t)}{\lambda_{n}} d t, \quad n \geq 1 .
$$

As $G(x, t)$ is the Green function of the problem (5), $-\int_{a}^{b} G(x, t)\left(\Phi_{n}^{\prime \prime}(t) / \lambda_{n}\right) d t$ is the value at $x$ of the function satisfying (3) whose second derivative is $\Phi_{n}^{\prime \prime} / \lambda_{n}$, that is, $\Phi_{n}(x) / \lambda_{n}$. This proves (49). As for (50), note simply that

$$
\begin{aligned}
\left\langle g, \Phi_{n}\right\rangle & =\int_{a}^{b} q(t) \frac{\delta G(x, t)}{\delta x} \Phi_{n}(t) d t \\
& =\frac{\delta \int_{a}^{b} q(t) G(x, t) \Phi_{n}(t) d t}{\delta x} \\
& =\frac{1}{\lambda_{n}} \frac{\delta \Phi_{n}(x)}{\delta x}, \quad n \geq 1 .
\end{aligned}
$$

Lemma 10. Assume that either $\left(a_{11} / a_{12}\right) \geq 0$ or $a_{12}=0$ and that either $-\left(a_{21} / a_{22}\right) \geq 0$ or $a_{22}=0$. Let $\lambda_{n}$ and $\Phi_{n}$ be defined as in Theorem 5. Let one suppose that $q(x)>0$ on $[a, b]$ and that it can be decomposed as $q(x)=c(x) / d(x)$ with $c(x)$ and $d(x)$ being nondecreasing functions. Depending on the values of $a_{11}, a_{12}, a_{21}$, and $a_{22}$, one has

(i) if $a_{12}=0$, then

$$
\Phi_{n}^{\prime 2}(a) \geq \frac{2 \lambda_{n} c(a)}{\int_{a}^{b} d(t) q(t) d t}, \quad n \geq 1
$$

(ii) if $a_{12} \neq 0$, then

$$
\Phi_{n}^{2}(a) \geq \frac{2 d(a)}{\int_{a}^{b} d(t) q(t) d t\left(1+\left(a_{11}^{2} / a_{12}^{2} q(a) \lambda_{n}\right)\right)}, \quad n \geq 1
$$

(iii) if $a_{22}=0$, then

$$
\Phi_{n}^{\prime 2}(b) \geq \frac{2 \lambda_{n}}{d(b) \int_{a}^{b}(q(t) / c(t)) d t}, \quad n \geq 1 ;
$$


(iv) if $a_{22} \neq 0$, then

$$
\begin{aligned}
& \Phi_{n}^{2}(b) \\
& \geq \frac{2}{c(b) \int_{a}^{b}(q(t) / c(t)) d t\left(1+\left(a_{21}^{2} / a_{22}^{2} q(b) \lambda_{n}\right)\right)},
\end{aligned}
$$

$$
n \geq 1 \text {. }
$$

Proof. First of all, let us note that $q(x)$ can always be decomposed in the mentioned manner, given that $q(x)>0$ and therefore $\ln q(x)$ exists in $[a, b]$ and can be expressed as the difference between two increasing functions $A(x)$ and $B(x)$; that is,

$$
\ln q(x)=A(x)-B(x) .
$$

which, taking exponentials, becomes

$$
\begin{aligned}
q(x) & =\exp \ln q(x)=\exp (A(x)-B(x)) \\
& =\frac{\exp A(x)}{\exp B(x)}=\frac{c(x)}{d(x)},
\end{aligned}
$$

with $c(x)$ and $d(x)$ nondecreasing. Now let us consider the functional $F$ defined by

$$
F\left(\Phi_{n}, x\right)=\Phi_{n}^{2}(x)+\frac{\left(\Phi_{n}^{\prime}(x)\right)^{2}}{\lambda_{n} q(x)}, \quad x \in[a, b] .
$$

Using integration by parts and the fact that $\Phi_{n}$ is orthonormal with respect to the norm (11) it is easy to prove that

$$
\begin{aligned}
\int_{a}^{b} q(x) F\left(\Phi_{n}, x\right) d x & \\
= & \int_{a}^{b} q(x) \Phi_{n}^{2}(x) d x+\int_{a}^{b} \frac{\left(\Phi_{n}^{\prime}(x)\right)^{2}}{\lambda_{n}} d x \\
= & 2 \int_{a}^{b} q(x) \Phi_{n}^{2}(x) d x \\
& +\frac{1}{\lambda_{n}}\left(\Phi_{n}^{\prime}(b) \Phi_{n}(b)-\Phi_{n}^{\prime}(a) \Phi_{n}(a)\right) \\
= & 2+\frac{1}{\lambda_{n}}\left(\Phi_{n}^{\prime}(b) \Phi_{n}(b)-\Phi_{n}^{\prime}(a) \Phi_{n}(a)\right) .
\end{aligned}
$$

From (3), (61), and the hypothesis one gets

$$
\int_{a}^{b} q(x) F\left(\Phi_{n}, x\right) d x \geq 2
$$

And given that $c(x)$ and $d(x)$ are increasing on $[a, b]$ and that $\Phi_{n}(x)$ verifies (28) for $\lambda=\lambda_{n}$, one has

$$
\begin{array}{r}
\left(c(x) F\left(\Phi_{n}, x\right)\right)^{\prime}=\left(c(x) \Phi_{n}^{2}(x)+d(x) \frac{\left(\Phi_{n}^{\prime}(x)\right)^{2}}{\lambda_{n}}\right)^{\prime} \\
=c^{\prime}(x) \Phi_{n}^{2}(x)+d^{\prime}(x) \frac{\left(\Phi_{n}^{\prime}(x)\right)^{2}}{\lambda_{n}} \geq 0, \\
x \in] a, b[;
\end{array}
$$

that is, $c(x) F\left(\Phi_{n}, x\right)$ is increasing on $] a, b[$, and

$$
\begin{aligned}
&\left(\frac{F\left(\Phi_{n}, x\right)}{d(x)}\right)^{\prime}=\left(\frac{\Phi_{n}^{2}(x)}{d(x)}+\frac{\left(\Phi_{n}^{\prime}(x)\right)^{2}}{\lambda_{n} c(x)}\right)^{\prime} \\
&=-d^{\prime}(x) \frac{\Phi_{n}^{2}(x)}{d^{2}(x)}-c^{\prime}(x) \frac{\left(\Phi_{n}^{\prime}(x)\right)^{2}}{\lambda_{n} c^{2}(x)} \leq 0, \\
&x \in] a, b[
\end{aligned}
$$

that is, $F\left(\Phi_{n}, x\right) / d(x)$ is decreasing on $] a, b[$. The application of (60), (63), and (64) to (62) leads to

$$
\begin{aligned}
& F\left(\Phi_{n}, x\right)\left\{c(x) \int_{a}^{x} \frac{q(t)}{c(t)} d t+\frac{1}{d(x)} \int_{x}^{b} d(t) q(t) d t\right\} \\
& \geq \int_{a}^{x} c(t) F\left(\Phi_{n}, t\right) \frac{q(t)}{c(t)} d t \\
& \quad+\int_{x}^{b} \frac{F\left(\Phi_{n}, t\right)}{d(t)} d(t) q(t) d t \\
& \geq \int_{a}^{b} q(x) F\left(\Phi_{n}, x\right) d x \geq 2, \quad n \geq 1 .
\end{aligned}
$$

From (3), (60), and (65) it is straightforward to obtain (54)(57).

Lemma 11. Let $\lambda_{n}$ and $\Phi_{n}$ be defined as in Theorem 5. Then one has

$$
\lambda_{n} \leq \frac{(n+C)^{2} \pi^{2}}{\left(\int_{a}^{b} \min (1, q(x)) d x\right)^{2}}=M(n+C)^{2}, \quad n \geq 1,
$$

with

$$
C= \begin{cases}0, & \text { if } \frac{a_{11}}{a_{12}}=\frac{a_{21}}{a_{22}} \text { or } a_{22}=a_{12}=0 ; \\ -\frac{1}{2}, & \text { if } a_{12}=0, \quad a_{22} \neq 0, \frac{-a_{21}}{a_{22}} \leq 0 ; \\ -\frac{1}{2}, & \text { if } a_{22}=0, \quad a_{12} \neq 0, \frac{-a_{11}}{a_{12}} \geq 0 ; \\ -1, & \text { else. }\end{cases}
$$

Proof. From [23, Theorem 8], if we define the function angle $\theta_{n}(x)$ by

$$
\tan \theta_{n}(x)=\frac{\sin \theta_{n}(x)}{\cos \theta_{n}(x)}=\frac{\Phi_{n}^{\prime}(x)}{A \Phi_{n}(x)}
$$

where $A>0$ is a real constant, then $\theta_{n}(x)$ satisfies the equation

$$
\theta_{n}^{\prime}(x)=-A \sin ^{2} \theta(x)-\frac{\lambda_{n} q(x)}{A} \cos ^{2} \theta(x),
$$


being, therefore, a decreasing function. Let us fix $]-(\pi / 2)$, $(\pi / 2)$ [as the range of the arctan function. In that case,

(i) if $a_{12}=0$ we will set $\theta_{n}(a)=\pi / 2$. Else, we will set $\theta_{n}(a)=\arctan \left(-a_{11} / a_{12} A\right)$;

(ii) if $a_{22}=0$ we will define $\vartheta_{n}(b)=-(\pi / 2)$. Else,

(a) if $-a_{11} / a_{12} \geq-a_{21} / a_{22}$ we will define $\vartheta_{n}(b)=$ $\arctan \left(-a_{21} / a_{22} A\right)$

(b) we will define $\vartheta_{n}(b)=-\pi+\arctan \left(-a_{21} / a_{22} A\right)$.

With this in mind, we will also define

$$
K(A)=\frac{\theta_{n}(a)-\vartheta_{n}(b)}{\pi}-1
$$

$K(A)$ is a constant which depends only on $A$ and the boundary conditions (3) and is related to the angle distance between $a$ and $b$ due to (3). Since $\Phi_{n}(x)$ is a function satisfying (3) with one more zero in $] a, b\left[\right.$ than $\Phi_{n-1}(x)$, integrating (69) and taking (70) into account we obtain

$$
\begin{aligned}
(n+ & K(A)) \pi \\
= & -\int_{a}^{b} \theta_{n}^{\prime}(x) d x=A \int_{a}^{b} \sin ^{2} \theta(x) d x \\
& +\int_{a}^{b} \frac{\lambda_{n} q(x)}{A} \cos ^{2} \theta(x) d x .
\end{aligned}
$$

Taking $A=\sqrt{\lambda_{n}}$ in (71) one has

$$
\begin{aligned}
& \left(n+K\left(\sqrt{\lambda_{n}}\right)\right) \pi \\
& =\sqrt{\lambda_{n}}\left(\int_{a}^{b} \sin ^{2} \theta(x) d x+\int_{a}^{b} q(x) \cos ^{2} \theta(x) d x\right) \\
& \geq \sqrt{\lambda_{n}} \int_{a}^{b} \min (1, q(x)) d x .
\end{aligned}
$$

The problem for (72) to be used to get upper bounds for $\lambda_{n}$ is the dependence of $K\left(\sqrt{\lambda_{n}}\right)$ on $\sqrt{\lambda_{n}}$. We can eliminate it by obtaining an upper bound for $K\left(\sqrt{\lambda_{n}}\right)$. Accordingly we will define

$$
C= \begin{cases}0, & \text { if } \frac{a_{11}}{a_{12}}=\frac{a_{21}}{a_{22}} \text { or } a_{22}=a_{12}=0 \\ -\frac{1}{2}, & \text { if } a_{12}=0, \quad a_{22} \neq 0, \frac{-a_{21}}{a_{22}} \leq 0 \\ -\frac{1}{2}, & \text { if } a_{22}=0, \quad a_{12} \neq 0, \frac{-a_{11}}{a_{12}} \geq 0 \\ -1, & \text { else. }\end{cases}
$$

From (70), (72), and (73) one yields

$$
(n+C) \pi \geq \sqrt{\lambda_{n}} \int_{a}^{b} \min (1, q(x)) d x .
$$

Taking squares in (74) one finally gets to (66).
Finally, by (25) and Lemmas 9-11 we are in a position to prove the next theorem, which allows a straightforward application of the method based on Theorem 8 .

Theorem 12. Let $\lambda_{n}$ and $\Phi_{n}$ be defined as in Theorem 5. Let $c(x)$ and $d(x)$ be defined as in Lemma 10. Let $M$ and $C$ be defined as in Lemma 11. Assume that there is a nontrivial solution $y(x)$ of (2) that satisfies (3) at some $a^{\prime}, b^{\prime}$ interior to $[a, b]$. Assume also that $a, b$ satisfy any of conditions (12) of Lemma 3, that either $\left(a_{11} / a_{12}\right) \geq 0$ or $a_{12}=0$, and that either $-\left(a_{21} / a_{22}\right) \geq 0$ or $a_{22}=0$. Then the following inequalities hold.

(i) If $a_{12}=0$ then

$$
\begin{array}{r}
\left\|L^{k}\left\{\frac{\delta G(a, t)}{\delta x}\right\}\right\|_{2}^{2} \\
>\frac{2 c(a)}{\int_{a}^{b} d(t) q(t) d t}\left(1+\frac{1}{(4 k+1) M^{2 k+1}(2+C)^{4 k+1}}\right), \\
k \geq 1 .
\end{array}
$$

Otherwise, if $N_{a}$ is the first integer greater than 1 such that $M\left(N_{a}+C\right)^{2} \geq a_{11}^{2} / a_{12}^{2} q(a)$, then

$$
\begin{aligned}
& \left\|L^{k}\{G(a, t)\}\right\|_{2}^{2} \\
& >\frac{2 d(a)}{\int_{a}^{b} d(t) q(t) d t} \\
& \quad \times\left(\frac{1}{1+\left(a_{11}^{2} / a_{12}^{2} q(a)\right)}\right. \\
& \quad+\sum_{n=2}^{N_{a}-1}\left(M^{2 k+1}(n+C)^{4 k+2}\right. \\
& \left.\quad \times \frac{1}{2 M^{2 k+2}(4 k+3)\left(N_{a}+C\right)^{4 k+3}}\right), \quad k \geq 1 .
\end{aligned}
$$

(ii) If $a_{22}=0$ then

$$
\begin{aligned}
&\left\|L^{k}\left\{\frac{\delta G(b, t)}{\delta x}\right\}\right\|_{2}^{2} \\
&> \frac{2}{d(b) \int_{a}^{b}(q(t) / c(t)) d t} \\
& \times\left(1+\frac{1}{(4 k+1) M^{2 k+1}(2+C)^{4 k+1}}\right),
\end{aligned}
$$


Otherwise, if $N_{b}$ is the first integer greater than 1 such that $M\left(N_{b}+C\right)^{2} \geq a_{21}^{2} / a_{22}^{2} q(b)$, then

$$
\begin{aligned}
& \left\|L^{k}\{G(b, t)\}\right\|_{2}^{2} \\
& >\frac{2}{c(b) \int_{a}^{b}(q(t) / c(t)) d t} \\
& \quad \times\left(\frac{1}{1+\left(a_{21}^{2} / a_{22}^{2} q(b)\right)}\right. \\
& \quad+\sum_{n=2}^{N_{b}-1}\left(M^{2 k+1}(n+C)^{4 k+2}\right. \\
& \left.\quad \times\left(M(n+C)^{2}+\left(a_{21}^{2} / a_{22}^{2} q(b)\right)\right)\right)^{-1} \\
& \left.\quad+\frac{1}{2 M^{2 k+2}(4 k+3)\left(N_{b}+C\right)^{4 k+3}}\right), \quad k \geq 1 .
\end{aligned}
$$

Proof. Let us focus first on (75). From (25), the fact that $\lambda_{1}<1$ (from the hypothesis) and Lemmas 9-10 it is straightforward to show that

$$
\begin{aligned}
& \left\|L^{k}\left\{\frac{\delta G(a, t)}{\delta x}\right\}\right\|_{2}^{2} \\
& >\frac{2 c(a)}{\int_{a}^{b} d(t) q(t) d t}\left(1+\sum_{n=2}^{\infty} \frac{1}{\lambda_{n}^{2 k+1}}\right), \quad k \geq 1 .
\end{aligned}
$$

On the other hand, from (66) one has

$$
\sum_{n=2}^{\infty} \frac{1}{\lambda_{n}^{2 k+1}} \geq \frac{1}{M^{2 k+1}} \sum_{n=2}^{\infty} \frac{1}{(n+C)^{4 k+2}}, \quad k \geq 1 .
$$

But given that

$$
\begin{aligned}
\sum_{n=2}^{\infty} \frac{1}{(n+C)^{4 k+2}} & \geq \int_{2}^{\infty} \frac{d x}{(x+C)^{4 k+2}} \\
& =\frac{1}{(4 k+1)(2+C)^{4 k+1}},
\end{aligned}
$$

from (79)-(81) one gets (75).

As for (76), again from (25) and Lemmas 9-10 it is straightforward to show that

$$
\begin{aligned}
& \left\|L^{k}\{G(a, t)\}\right\|_{2}^{2} \\
& >\frac{2 d(a)}{\int_{a}^{b} d(t) q(t) d t} \\
& \quad \times\left(\frac{1}{\lambda_{1}^{2}+\lambda_{1}\left(a_{11}^{2} / a_{12}^{2} q(a)\right)}\right. \\
& \left.\quad+\sum_{n=2}^{\infty} \frac{1}{\lambda_{n}^{2 k}\left(\lambda_{n}^{2}+\lambda_{n}\left(a_{11}^{2} / a_{12}^{2} q(a)\right)\right)}\right), \\
& k \geq 1 .
\end{aligned}
$$

On the other hand, from (66) one has

$$
\begin{aligned}
& \sum_{n=2}^{\infty} \frac{1}{\lambda_{n}^{2 k}\left(\lambda_{n}^{2}+\lambda_{n}\left(a_{11}^{2} / a_{12}^{2} q(a)\right)\right)} \\
& \geq \sum_{n=2}^{\infty}\left(M^{2 k+1}(n+C)^{4 k+2}\right. \\
& \left.\quad \times\left(M(n+C)^{2}+\frac{a_{11}^{2}}{a_{12}^{2} q(a)}\right)\right)^{-1},
\end{aligned}
$$

$$
k \geq 1 \text {. }
$$

From the definition of $N_{a}$, it is clear that

$$
\begin{gathered}
\frac{1}{M^{2 k+1}(n+C)^{4 k+2}\left(M(n+C)^{2}+\left(a_{11}^{2} / a_{12}^{2} q(a)\right)\right)} \\
\geq \frac{1}{2 M^{2 k+2}(n+C)^{4 k+4}}, \quad n \geq N_{a}, \quad k \geq 1 .
\end{gathered}
$$

And given that

$$
\begin{aligned}
\sum_{N_{a}}^{\infty} \frac{1}{(n+C)^{4 k+4}} & \geq \int_{N_{a}}^{\infty} \frac{d x}{(x+C)^{4 k+4}} \\
& =\frac{1}{(4 k+3)\left(N_{a}+C\right)^{4 k+3}},
\end{aligned}
$$

from (82)-(85) and the fact that $\lambda_{1}<1$ by the hypothesis, one obtains (76). The proofs of (77) and (78) are very similar mutatis mutandi.

Remark 13. It is possible to improve the results of Theorem 12 by using better lower bounds for $\Phi_{n}^{2}(a), \Phi_{n}^{2}(b), \Phi_{n}^{\prime 2}(a)$, and $\Phi_{n}^{\prime 2}(b)$ than the ones displayed in Lemma 10 and better upper bounds for $\lambda_{n}$ than the one presented in Lemma 11 or calculating more terms in the sums of (75)-(78) before using the integrals to get lower bounds for the remainders of the series.

\section{Bounds for the Distance between $a$ and $b$ Based on Concrete Values of $L^{k} f(x)$}

This section will elaborate on the results of Section 2 in order to obtain upper and lower bounds for the distance between the extremes $a, b$ in a similar way as it was done in [1]. A critical condition for that is the positivity of the Green function $G(x, t)$, which is ensured under certain boundary conditions by the next lemma. 
Lemma 14. Let $a, b$ be any real numbers such that $a \neq b$ and $a, b \in\left[a^{\prime}, b^{\prime}\right]$. If the boundary conditions (3) verify any of the following hypotheses for $a^{\prime}, b^{\prime}$,

$$
\begin{gathered}
a_{12}=a_{22}=0, \\
a_{12}=0, \quad 1+\frac{a_{21}}{a_{22}}\left(b^{\prime}-a^{\prime}\right)>0, \\
1-\frac{a_{11}}{a_{12}}\left(b^{\prime}-a^{\prime}\right)>0, \quad a_{22}=0, \\
\frac{a_{21}}{a_{22}}>\frac{a_{11}}{a_{12}}, \quad \frac{a_{21}}{a_{22}}-\frac{a_{11}}{a_{12}}-\frac{a_{21}}{a_{22}} \frac{a_{11}}{a_{12}}\left(b^{\prime}-a^{\prime}\right)>0, \\
\text { either } 1+\frac{a_{21}}{a_{22}}\left(b^{\prime}-a^{\prime}\right)>0 \quad \text { or } 1-\frac{a_{11}}{a_{12}}\left(b^{\prime}-a^{\prime}\right)>0,
\end{gathered}
$$

then $G_{a b}(x, t)$ is positive almost everywhere for $t \in[a, b]$ except if $x=a$ or $x=b$ in case (86), $x=a$ in case (87), and $x=b$ in case (88).

Proof. As was mentioned in the proof of Lemma 3, if we define $y_{1}(x)$ and $y_{2}(x)$ as the solutions of

$$
\begin{gathered}
y^{\prime \prime}=0, \quad a_{11} y\left(a^{\prime}\right)+a_{12} y^{\prime}\left(a^{\prime}\right)=0, \\
y^{\prime \prime}=0, \quad a_{21} y\left(b^{\prime}\right)+a_{22} y^{\prime}\left(b^{\prime}\right)=0,
\end{gathered}
$$

respectively, then we can represent $G_{a^{\prime} b^{\prime}}(x, t)$ as follows:

$$
G_{a^{\prime} b^{\prime}}(x, t)= \begin{cases}\frac{-y_{1}(x) y_{2}(t)}{y_{1}\left(a^{\prime}\right) y_{2}^{\prime}\left(a^{\prime}\right)-y_{1}^{\prime}\left(a^{\prime}\right) y_{2}\left(a^{\prime}\right)}, & x<t, \\ \frac{-y_{1}(t) y_{2}(x)}{y_{1}\left(a^{\prime}\right) y_{2}^{\prime}\left(a^{\prime}\right)-y_{1}^{\prime}\left(a^{\prime}\right) y_{2}\left(a^{\prime}\right)}, & x \geq t .\end{cases}
$$

We can divide the analysis of (91) in four cases.

(i) $a_{12}=0$ and $a_{22}=0$. In this case one has

$$
G_{a^{\prime} b^{\prime}}(x, t)= \begin{cases}\frac{\left(x-a^{\prime}\right)\left(t-b^{\prime}\right)}{a^{\prime}-b^{\prime}}, & x<t, \\ \frac{\left(t-a^{\prime}\right)\left(x-b^{\prime}\right)}{a^{\prime}-b^{\prime}}, & x \geq t,\end{cases}
$$

which is clearly positive almost everywhere for $t \in$ $\left[a^{\prime}, b^{\prime}\right]$ as long as $x \neq a^{\prime}$ and $x \neq b^{\prime}$. This yields condition (86).

(ii) $a_{12}=0$ and $a_{22} \neq 0$. In this case, one has

$$
G_{a^{\prime} b^{\prime}}(x, t)= \begin{cases}\frac{\left(x-a^{\prime}\right)\left(-\left(a_{21} / a_{22}\right)\left(t-b^{\prime}\right)+1\right)}{-\left(a_{21} / a_{22}\right)\left(a^{\prime}-b^{\prime}\right)+1}, & x<t, \\ \frac{\left(t-a^{\prime}\right)\left(-\left(a_{21} / a_{22}\right)\left(x-b^{\prime}\right)+1\right)}{-\left(a_{21} / a_{22}\right)\left(a^{\prime}-b^{\prime}\right)+1}, & x \geq t .\end{cases}
$$

From (93) it is clear that $G_{a^{\prime} b^{\prime}}(x, t)$ will be positive almost everywhere for $t \in\left[a^{\prime}, b^{\prime}\right]$ as long as $x \neq a^{\prime}$ and

$$
1+\frac{a_{21}}{a_{22}}\left(b^{\prime}-a^{\prime}\right)>0
$$

which gives condition (87)

(iii) $a_{12} \neq 0$ and $a_{22}=0$. In this case, one has

$$
\begin{aligned}
& G_{a^{\prime} b^{\prime}}(x, t)= \\
& \begin{cases}-\frac{\left(t-b^{\prime}\right)\left(-\left(a_{11} / a_{12}\right)\left(x-a^{\prime}\right)+1\right)}{-\left(a_{11} / a_{12}\right)\left(b^{\prime}-a^{\prime}\right)+1}, & x<t, \\
-\frac{\left(x-b^{\prime}\right)\left(-\left(a_{11} / a_{12}\right)\left(t-a^{\prime}\right)+1\right)}{-\left(a_{11} / a_{12}\right)\left(b^{\prime}-a^{\prime}\right)+1}, & x \geq t .\end{cases}
\end{aligned}
$$

From (95) it is clear that $G_{a^{\prime} b^{\prime}}(x, t)$ will be positive almost everywhere for $t \in\left[a^{\prime}, b^{\prime}\right]$ as long as $x \neq b^{\prime}$ and

$$
1-\frac{a_{11}}{a_{12}}\left(b^{\prime}-a^{\prime}\right)>0
$$

which gives condition (88).

(iv) $a_{12} \neq 0$ and $a_{22} \neq 0$. In this case, one has

$G_{a^{\prime} b^{\prime}}(x, t)$

$$
=\left\{\begin{array}{r}
\frac{\left(-\left(a_{21} / a_{22}\right)\left(t-b^{\prime}\right)+1\right)\left(-\left(a_{11} / a_{12}\right)\left(x-a^{\prime}\right)+1\right)}{\left(-\left(a_{11} / a_{12}\right)\left(b^{\prime}-a^{\prime}\right)+1\right)\left(a_{21} / a_{22}\right)-\left(a_{11} / a_{12}\right)}, \\
x<t, \\
\frac{\left(-\left(a_{21} / a_{22}\right)\left(x-b^{\prime}\right)+1\right)\left(-\left(a_{11} / a_{12}\right)\left(t-a^{\prime}\right)+1\right)}{\left(-\left(a_{11} / a_{12}\right)\left(b^{\prime}-a^{\prime}\right)+1\right)\left(a_{21} / a_{22}\right)-\left(a_{11} / a_{12}\right)}, \\
x \geq t .
\end{array}\right.
$$

From (97) it is clear that $G_{a^{\prime} b^{\prime}}(x, t)$ will be positive almost everywhere for $t \in\left[a^{\prime}, b^{\prime}\right]$ if $1-\left(a_{11} / a_{12}\right)\left(b^{\prime}-\right.$ $\left.a^{\prime}\right)>0,1-\left(a_{21} / a_{22}\right)\left(a^{\prime}-b^{\prime}\right)>0$, and $\left(-\left(a_{11} / a_{12}\right)\left(b^{\prime}-\right.\right.$ $\left.\left.a^{\prime}\right)+1\right)\left(a_{21} / a_{22}\right)-\left(a_{11} / a_{12}\right)>0$. Condition (89) is a consequence of this and the fact that $\left(-\left(a_{11} / a_{12}\right)\left(b^{\prime}-\right.\right.$ $\left.\left.a^{\prime}\right)+1\right)\left(a_{21} / a_{22}\right)-\left(a_{11} / a_{12}\right)>0$ and $1-\left(a_{11} / a_{12}\right)\left(b^{\prime}-\right.$ $\left.a^{\prime}\right)>0$ imply $1-\left(a_{21} / a_{22}\right)\left(a^{\prime}-b^{\prime}\right)>0$ and that $\left(-\left(a_{11} / a_{12}\right)\left(b^{\prime}-a^{\prime}\right)+1\right)\left(a_{21} / a_{22}\right)-\left(a_{11} / a_{12}\right)>0$ and $1-\left(a_{21} / a_{22}\right)\left(a^{\prime}-b^{\prime}\right)>0$ imply $1-\left(a_{11} / a_{12}\right)\left(b^{\prime}-a^{\prime}\right)>0$ (both proofs are straightforward and are left to the reader).

To complete the theorem it suffices to prove that if (86)-(89) are met by $a^{\prime}, b^{\prime}$, they will also be met by any other extremes $a$, $b$ such that $[a, b] \subset\left[a^{\prime}, b^{\prime}\right]$. This is straightforward in the case of conditions $1-\left(a_{11} / a_{12}\right)\left(b^{\prime}-a^{\prime}\right)>0$ and $1+\left(a_{21} / a_{22}\right)\left(b^{\prime}-\right.$ $\left.a^{\prime}\right)>0$. In the case of the condition $\left(a_{21} / a_{22}\right)-\left(a_{11} / a_{12}\right)-$ $\left(a_{21} / a_{22}\right)\left(a_{11} / a_{12}\right)\left(b^{\prime}-a^{\prime}\right)>0$ simply note that

$$
h(x)=\frac{a_{21}}{a_{22}}-\frac{a_{11}}{a_{12}}-\frac{a_{21}}{a_{22}} \frac{a_{11}}{a_{12}} x, \quad x \in\left[0, b^{\prime}-a^{\prime}\right],
$$


is a linear function positive at the extremes 0 and $b^{\prime}-a^{\prime}$ by the hypothesis, and therefore it must be positive for any $b-a \in$ $\left[0, b^{\prime}-a^{\prime}\right]$.

Remark 15. Conditions (86)-(89) imply conditions (12) of Lemma 3. In consequence they will replace the latter in the rest of results of this section.

Once the conditions for the positivity of the Green function have been determined, we can proceed with the key results of this method.

Lemma 16. Assume that $\left[a^{\prime}, b^{\prime}\right] \subset\left[a^{\prime \prime}, b^{\prime \prime}\right]$ are enclosed intervals such that $\left[a^{\prime}, b^{\prime}\right] \neq\left[a^{\prime \prime}, b^{\prime \prime}\right]$ and that any of conditions (86)-(89) are met in $\left[a^{\prime \prime}, b^{\prime \prime}\right]$. Assume also that $f(x)$ is positive almost everywhere on $\left[a^{\prime \prime}, b^{\prime \prime}\right]$ and that $x \in\left[a^{\prime}, b^{\prime}\right]$ is such that $G(x, t)$ is not identically zero. Then the operator $L$ satisfies

$$
L_{a^{\prime}, b^{\prime}} f(x)<L_{a^{\prime \prime}, b^{\prime \prime}} f(x)<\max \left\{L_{a^{\prime \prime}, b^{\prime \prime}} f(t), t \in\left[a^{\prime \prime}, b^{\prime \prime}\right]\right\} .
$$

Proof. Since $q(x)$ and $f(x)$ are positive almost everywhere on $\left[a^{\prime \prime}, b^{\prime \prime}\right]$ by hypothesis, and $G_{a^{\prime} b^{\prime}}$ and $G_{a^{\prime \prime}} b^{\prime \prime}$ are also positive almost everywhere on $\left[a^{\prime}, b^{\prime}\right]$ and $\left[a^{\prime \prime}, b^{\prime \prime}\right]$, respectively, by the hypothesis and Lemma 14 , it suffices to prove that

$$
G_{a^{\prime} b^{\prime}}(x, t) \leq G_{a^{\prime \prime} b^{\prime \prime}}(x, t)
$$

for $(x, t) \in\left[a^{\prime}, b^{\prime}\right] \times\left[a^{\prime}, b^{\prime}\right]$. We will do it only for the case $a_{12}, a_{22} \neq 0$ (the proof of the rest of cases is very similar), for which the Green function is given by (97). If we denote by $W$ the expression $\left(-\left(a_{11} / a_{12}\right)\left(b^{\prime}-a^{\prime}\right)+1\right)\left(a_{21} / a_{22}\right)-\left(a_{11} / a_{12}\right)$, a routine calculation yields

$$
\begin{aligned}
& \frac{\delta G_{a^{\prime} b^{\prime}}(x, t)}{\delta a^{\prime}} \\
& =\left\{\begin{array}{cc}
-\left(\frac{a_{11}}{a_{12} W}\right)^{2}\left(-\frac{a_{21}}{a_{22}}\left(x-b^{\prime}\right)+1\right) & \\
\times\left(-\frac{a_{21}}{a_{22}}\left(t-b^{\prime}\right)+1\right), & x \geq t, \\
-\left(\frac{a_{11}}{a_{12} W}\right)^{2}\left(-\frac{a_{21}}{a_{22}}\left(t-b^{\prime}\right)+1\right) & \\
\times\left(-\frac{a_{21}}{a_{22}}\left(x-b^{\prime}\right)+1\right), & x<t .
\end{array}\right.
\end{aligned}
$$

From (89), which is the hypothesis applicable if $a_{12}, a_{22} \neq 0$, one has $\delta G_{a^{\prime} b^{\prime}}(x, t) / \delta a^{\prime}<0$ if $a_{11} \neq 0$ and $\delta G_{a^{\prime} b^{\prime}}(x, t) / \delta a^{\prime} \equiv 0$ if $a_{11}=0$. In other words, fixing $x, t$, and $b^{\prime}$, either $G_{a^{\prime} b^{\prime}}(x, t)$ increases $\left(a_{11} \neq 0\right)$ or $G_{a^{\prime} b^{\prime}}(x, t)$ stays constant $\left(a_{11}=0\right)$ as $a^{\prime}$ decreases.

In a similar manner we can show that $\delta G_{a^{\prime} b^{\prime}}(x, t) / \delta b^{\prime} \geq 0$; that is, fixing $x, t$, and $a^{\prime}$, either $G_{a^{\prime} b^{\prime}}(x, t)$ increases $\left(a_{21} \neq 0\right)$ or $G_{a^{\prime} b^{\prime}}(x, t)$ stays constant $\left(a_{21}=0\right)$ as $b^{\prime}$ increases. This proves (100) and the lemma.
Lemma 17. Assume that any of conditions (86)-(89) holds in $[a, b]$. If
(i) either $a_{12}=a_{22}=0$,
(ii) or $a_{12}=0$ and $\left(a_{21} / a_{22}\right)>0$,
(iii) or $a_{22}=0$ and $\left(a_{11} / a_{12}\right)<0$,
(iv) or $\left(a_{11} / a_{12}\right)<0<\left(a_{21} / a_{22}\right)$,

then one has

$$
\max \{G(x, t), x \in[a, b]\}=G(t, t), \quad t \in[a, b] .
$$

Proof. We will only prove the last case (the proof of the other cases is very similar). Thus, from (97) it follows that, fixing $t, G(x, t)$ is a function increasing with $x$ for $x \leq t$ and decreasing with $x$ for $t<x$. Therefore it will have a maximum at $x=t$, which is exactly (102).

Theorem 18. Let one suppose that there exists a nontrivial solution $y(x)$ of (2) that satisfies (3) at $a, b$. Let $y_{\max }$ be defined by $y_{\max }=\max \{y(x), x \in[a, b]\}$. Assume that any of conditions (86)-(89) holds in extremes $a^{\prime}, b^{\prime}$ such that $[a, b] \subset$ $\left[a^{\prime}, b^{\prime}\right]$. Assume also that $f>y$ almost everywhere on $[a, b]$ and $f>0$ almost everywhere on $\left[a^{\prime}, b^{\prime}\right]$. Then

$$
L_{a^{\prime}, b^{\prime}}^{k} f(x)>y(x), \quad k \geq 1, x \in[a, b] .
$$

Moreover,

(i) if $\left(a_{11} / a_{12}\right) \geq 0$ and either $a_{22}=0$ or $\left(a_{21} / a_{22}\right)>0$, then

$$
L_{a^{\prime}, b^{\prime}}^{k} f\left(a^{\prime}\right)>y_{\max }=y(a), \quad k \geq 1 ;
$$

(ii) if $\left(a_{21} / a_{22}\right) \leq 0$ and either $a_{12}=0$ or $\left(a_{11} / a_{12}\right)<0$, then

$$
L_{a^{\prime}, b^{\prime}}^{k} f\left(b^{\prime}\right)>y_{\max }=y(b), \quad k \geq 1 ;
$$

(iii) if any of the conditions of Lemma 17 are met, then

$$
\int_{a^{\prime}}^{b^{\prime}} q(t) G_{a^{\prime} b^{\prime}}(t, t) L_{a^{\prime} b^{\prime}}^{k-1} f(t) d t>y_{\max }, \quad k \geq 1 .
$$

Proof. From the hypothesis and Lemma 14, $G_{a b}(x, t)$ is either positive almost everywhere or identically zero on $t \in[a, b]$, depending on the value of $x$. In consequence we can apply Lemma 1 recursively to yield

$$
L_{a, b}^{k} f(x) \geq L_{a, b}^{k} y(x)=y(x), \quad k \geq 1 .
$$

And given that $f(x)>0$ almost everywhere on $\left[a^{\prime}, b^{\prime}\right]$, from Lemma 16 and (107) one gets (103). Equations (104) and (105) are consequences of $y$ and $L_{a^{\prime}, b^{\prime}}^{k} f$ having maxima at $a$ and $a^{\prime}$, in the first case, and $b$ and $b^{\prime}$, in the second case, respectively. As for (106), it results from (103) and Lemma 17. 
Theorem 19. Let one suppose that there exists a nontrivial solution $y(x)$ of (2) that satisfies (3) at $a, b$. Let $y_{\max }$ be defined by $y_{\max }=\max \{y(x), x \in[a, b]\}$. Assume that any of conditions (86)-(89) holds at $a, b$ and that the extremes $a^{\prime}, b^{\prime}$ are such that $\left[a^{\prime}, b^{\prime}\right] \subset[a, b]$. Assume also that $0<f(x)<y(x)$ almost everywhere on $\left[a^{\prime}, b^{\prime}\right]$. Then

$$
L_{a^{\prime}, b^{\prime}}^{k} f(x)<y(x), \quad k \geq 1, x \in\left[a^{\prime}, b^{\prime}\right] .
$$

Moreover,

(i) if $\left(a_{11} / a_{12}\right) \geq 0$ and either $a_{22}=0$ or $\left(a_{21} / a_{22}\right)>0$, then

$$
L_{a^{\prime}, b^{\prime}}^{k} f\left(a^{\prime}\right)<y_{\max }=y(a), \quad k \geq 1 ;
$$

(ii) if $\left(a_{21} / a_{22}\right) \leq 0$ and either $a_{12}=0$ or $\left(a_{11} / a_{12}\right)<0$, then

$$
L_{a^{\prime}, b^{\prime}}^{k} f\left(b^{\prime}\right)<y_{\max }=y(b), \quad k \geq 1 ;
$$

(iii) otherwise

$$
\max \left\{L_{a^{\prime}, b^{\prime}}^{k} f(x), x \in\left[a^{\prime}, b^{\prime}\right]\right\}<y_{\max }, \quad k \geq 1 .
$$

Proof. Applying Lemmas 1 and 16 recursively one has

$$
\begin{aligned}
y_{\max } & \geq y(x)=L_{a, b}^{k} y(x)>L_{a^{\prime}, b^{\prime}}^{k} y(x) \\
& \geq L_{a^{\prime}, b^{\prime}}^{k} f(x), \quad k \geq 1, \quad x \in\left[a^{\prime}, b^{\prime}\right] .
\end{aligned}
$$

This gives (108) and also (111). As before, (109) and (110) are consequences of $y$ and $L_{a^{\prime}, b^{\prime}}^{k} f$ having maxima at $a$ and $a^{\prime}$, in the first case, and $b$ and $b^{\prime}$, in the second case, respectively.

Corollary 20. Let one suppose that there exists a nontrivial solution $y(x)$ of (2) that satisfies (3) at $a, b$. Assume that any of conditions (86)-(89) holds at the extremes $a^{\prime}, b^{\prime}$.

(i) If $\left(a_{11} / a_{12}\right) \geq 0$ and either $a_{22}=0$ or $\left(a_{21} / a_{22}\right)>0$, then

$$
\begin{gathered}
L_{a^{\prime}, b^{\prime}}^{k}\{1\}\left(a^{\prime}\right)>1, \quad[a, b] \subset\left[a^{\prime}, b^{\prime}\right], k \geq 1, \\
L_{a^{\prime}, b^{\prime}}^{k}\left\{\frac{b^{\prime}-x}{b^{\prime}-a^{\prime}}\right\}\left(a^{\prime}\right)<1, \quad\left[a^{\prime}, b^{\prime}\right] \subset[a, b], k \geq 1 .
\end{gathered}
$$

(ii) If $\left(a_{21} / a_{22}\right) \leq 0$ and either $a_{12}=0$ or $\left(a_{11} / a_{12}\right)<0$, then

$$
\begin{gathered}
L_{a^{\prime}, b^{\prime}}^{k}\{1\}\left(b^{\prime}\right)>1, \quad[a, b] \subset\left[a^{\prime}, b^{\prime}\right], k \geq 1, \\
L_{a^{\prime}, b^{\prime}}^{k}\left\{\frac{x-a^{\prime}}{b^{\prime}-a^{\prime}}\right\}\left(b^{\prime}\right)<1, \quad\left[a^{\prime}, b^{\prime}\right] \subset[a, b], k \geq 1 .
\end{gathered}
$$

(iii) If any of the conditions of Lemma 17 are met and $c$ is any real number in $] a^{\prime}, b^{\prime}[$, then

$\int_{a^{\prime}}^{b^{\prime}} q(t) G_{a^{\prime} b^{\prime}}(t, t) L_{a^{\prime}, b^{\prime}}^{k-1}\{1\}(t) d t>1, \quad[a, b] \subset\left[a^{\prime}, b^{\prime}\right]$,

$$
k \geq 1
$$

$$
L_{a^{\prime}, b^{\prime}}^{k} g(c)<1, \quad\left[a^{\prime}, b^{\prime}\right] \subset[a, b], k \geq 1,
$$

where $g$ is defined by

$$
g(x)= \begin{cases}\frac{x-a^{\prime}}{c-a^{\prime}} & x \in\left[a^{\prime}, c\right], \\ \frac{b^{\prime}-x}{b^{\prime}-c} & x \in\left[c, b^{\prime}\right] .\end{cases}
$$

Proof. Equations (113), (115), and (117) can be easily obtained by setting $f(x) \equiv y_{\max }$ in (104)-(106). In turn, (114) can be obtained by setting $f(x) \equiv y\left(a^{\prime}\right)\left(\left(b^{\prime}-x\right) /\left(b^{\prime}-a^{\prime}\right)\right)$ (with $y\left(a^{\prime}\right)>0$ ) in (108) and taking into account that $y$ is concave (its second derivative is negative almost everywhere on $[a, b]$ since $q(x)$ and $y(x)$ are positive almost everywhere on $[a, b]$ due to (86)-(89)). Equations (116) and (118) can also be obtained in a similar manner.

Remark 21. Corollary 1 of [1] is a particular case (disfocality) of Corollary 20, (115)-(116).

Theorems 18 and 19 and Corollary 20 provide conditions to assess if there exists a solution $y(x)$ satisfying (3) at extremes inner or outer to $[a, b]$. However, at least in the way they have been presented, they do not allow determining if there exists a solution satisfying (3) at exactly the extremes $a$, $b$. That will be the purpose of the next theorems.

Theorem 22. Let one suppose that there exists a nontrivial solution $y(x)$ of (2) that satisfies (3) at $a, b$. Let $y_{\max }$ be defined by $y_{\max }=\max \{y(x), x \in[a, b]\}$. Assume that any of conditions (86)-(89) holds at $a, b$. Let $f(x)$ be such that $f(x)>$ $y(x)$ almost everywhere on $[a, b]$ and let $b_{k}$ be a sequence of real values whose definition depends on the boundary conditions (3) as follows:

(i) if $\left(a_{11} / a_{12}\right) \geq 0$ and either $a_{22}=0$ or $\left(a_{21} / a_{22}\right)>0$, then

$$
L_{a, b_{k}}^{k} f(a)=y(a), \quad k \geq 1
$$

(ii) if $\left(a_{21} / a_{22}\right) \leq 0$ and either $a_{12}=0$ or $\left(a_{11} / a_{12}\right)<0$, then

$$
L_{a, b_{k}}^{k} f\left(b_{k}\right)=y(b), \quad k \geq 1
$$

(iii) if any of the conditions of Lemma 17 are met, then

$$
\int_{a}^{b_{k}} q(t) G_{a b_{k}}(t, t) L_{a, b_{k}}^{k-1} f(t) d t=y_{\max }, \quad k \geq 1 ;
$$


with $f>0$ almost everywhere on $\left[a, b_{k}\right]$. Then $b_{k}<b$ for $k \geq 1$ and $\left\{b_{k}\right\}$ tends to $b$ as $k \rightarrow \infty$.

Proof. From the fact that $f(x)>y(x)$ almost everywhere on $[a, b]$ and $f(x)>0$ almost everywhere on $\left[a, b_{k}\right]$ and from $(104)-(106)$ and (120)-(122), it is clear that $\left[a, b_{k}\right] \subseteq[a, b]$ (i.e., $\left.a<b_{k} \leq b\right)$ for $k \geq 1$. Now, let us assume that $\left\{b_{k}\right\}$ does not have a limit in $b$. In that case there exist a $\delta>0$ and a subsequence $\left\{b_{k_{j}}\right\}$ of $\left\{b_{k}\right\}$ such that $b_{k_{j}}<b-\delta$. But then, from Theorem 5 and Lemma 16 one has

$$
\begin{aligned}
& \lim _{k_{j} \rightarrow \infty} L_{a, b_{k_{j}}}^{k_{j}} f(x) \leq \lim _{k_{j} \rightarrow \infty} L_{a, b-\delta}^{k_{j}} f(x)=0, \quad x \in[a, b-\delta], \\
& \lim _{k_{j} \rightarrow \infty} L_{a, b_{k_{j}}}^{k_{j}-1} f(x) \leq \lim _{k_{j} \rightarrow \infty} L_{a, b-\delta}^{k_{j}-1} f(x)=0, \quad x \in[a, b-\delta] .
\end{aligned}
$$

In both cases the convergence is uniform for $x \in[a, b-\delta]$. Therefore, for every $\epsilon>0$ there will exist infinitely many $b_{k_{j}}$ such that

$$
L_{a, b_{k_{j}}}^{k_{j}-1} f(x), L_{a, b_{k_{j}}}^{k_{j}} f(x)<\epsilon, \quad x \in\left[a, b_{k_{j}}\right]
$$

which contradicts (120) and (121) and, taking into account that

$$
\left|\int_{a}^{b_{k_{j}}} q(t) G(t, t) L_{a, b_{k_{j}}}^{k_{j}-1} f(t) d t\right|<\epsilon\left|\int_{a}^{b_{k_{j}}} q(t) G(t, t) d t\right|,
$$

also contradicts (122).

Theorem 23. Let one suppose that there exists a nontrivial solution $y(x)$ of (2) that satisfies (3) at $a, b$. Let $y_{\max }$ be defined by $y_{\max }=\max \{y(x), x \in[a, b]\}$. Let $b_{k}$ be a sequence of real values whose definition depends on the boundary conditions (3) as follows:

(i) if $\left(a_{11} / a_{12}\right) \geq 0$ and either $a_{22}=0$ or $\left(a_{21} / a_{22}\right)>0$, then

$$
L_{a, b_{k}}^{k} f_{a, b_{k}}(a)=y_{\max }, \quad k \geq 1
$$

(ii) if $\left(a_{21} / a_{22}\right) \leq 0$ and either $a_{12}=0$ or $\left(a_{11} / a_{12}\right)<0$, then

$$
L_{a, b_{k}}^{k} f_{a, b_{k}}\left(b_{k}\right)=y_{\max }, \quad k \geq 1 ;
$$

(iii) if any of the conditions of Lemma 17 are met, let one pick any $c \in\left[a, b_{k}\right]$ and set

$$
L_{a, b_{k}}^{k} f_{a, b_{k}}(c)=y_{\max }, \quad k \geq 1,
$$

where $f_{a, b_{k}}$ is a family of continuous functions on $\left[a, b_{k}\right]$ such that $0<f_{a, b_{k}}(x)<y(x)$ almost everywhere in $\left[a, b_{k}\right]$. Let one suppose that there exists a continuous function $f$ such that $0<$ $f(x)<f_{a, b_{k}}(x)$ almost everywhere in $\left[a, b_{k}\right]$. Assume that any of conditions (86)-(89) holds at $a, b_{k}$. Then $b_{k}>b$ for $k \geq 1$ and $\left\{b_{k}\right\}$ tends to $b$ as $k \rightarrow \infty$.
Proof. From the fact that $y(x)>f_{a, b_{k}}(x)>0$ almost everywhere on $\left[a, b_{k}\right],(109)-(111)$, and (126)-(128), it is clear that $\left[a, b_{k}\right] \supseteq[a, b]$ (i.e., $a<b \leq b_{k}$ ) for $k \geq 1$. Now, let us assume that $\left\{b_{k}\right\}$ does not have a limit in $b$. In that case there exist a $\delta>0$ and a subsequence $\left\{b_{k_{j}}\right\}$ of $\left\{b_{k}\right\}$ such that $b_{k_{j}}>b+\delta$. But then, from Theorem 5 and Lemmas 1 and 16, for those $x \in[a, b+\delta]$ such that $\Phi_{1}(x) \neq 0$, one has

$$
\begin{aligned}
& \lim _{k_{j} \rightarrow \infty} L_{a, b_{k_{j}}}^{k_{j}} f_{a, b_{k_{j}}}(x) \\
& \quad \geq \lim _{k_{j} \rightarrow \infty} L_{a, b_{k_{j}}}^{k_{j}} f(x) \geq \lim _{k_{j} \rightarrow \infty} L_{a, b+\delta}^{k_{j}} f(x)= \pm \infty,
\end{aligned}
$$

the sign depending on that of $\left\langle f, \Phi_{1}\right\rangle \Phi_{1}(x)$. Let us suppose that such a sign is positive (the negative case can be treated in the same manner). In that case for every $M>y_{\max }>0$ there will exist infinitely many $b_{k_{j}}$ such that

$$
L_{a, b_{k_{j}}}^{k_{j}} f_{a, b_{k_{j}}}(x)>M \text {. }
$$

Since the values at the left-hand side of (126)-(127) are upper bounds of $L_{a, b_{k_{j}}}^{k_{j}} f_{a, b_{k_{j}}}(x)$, (130) leads to a contradiction and proves the assertion.

Remark 24. Theorems 22 and 23 guarantee that, given $[a, b]$, one can determine if there exists a nontrivial solution $y(x)$ of (2) satisfying (3) at $a, b$ by calculating the sequences defined by (120)-(122) and (126)-(128) and checking if they converge to $b$.

\section{The Calculation of $L^{k} f(x)$}

A common key point of Sections 3 and 4 is the importance of the calculation of $L^{k} f(x)$ for different functions $f$. Bearing this in mind, our aim for this section is to find manners to facilitate such a calculation. That will be done with the following theorems, in all of which the internal product $\langle\cdot, \cdot\rangle$ is assumed to be calculated on the variable $t$.

Theorem 25. The operator L defined in (4) verifies

$$
L f(x)=\langle G(x, t), f(t)\rangle, \quad x \in[a, b] .
$$

Proof. It is straightforward from the definition of $L$ given in (4) and the definition of the internal product (10).

Theorem 26. If $a, b$ satisfy any of conditions (12), then the operator $L$ defined in (4) verifies

$$
\begin{array}{r}
L^{n+m+1} f(x)= \\
x \in[a, b], \quad n, m \geq 0 .
\end{array}
$$

And in particular

$$
L^{k+1} f(x)=\left\langle L^{k} G(x, t), f(t)\right\rangle, \quad x \in[a, b], k \geq 0 .
$$


Proof. From (131) one has

$$
\begin{array}{r}
L^{n+m+1} f(x)= \\
x \in\left[G(x, t), L^{n+m} f(t)\right\rangle, \quad n, m \geq 0 .
\end{array}
$$

From the hypothesis and Lemma $3, L$ is self-adjoint. We can apply that property recursively to (134) to obtain

$$
\begin{aligned}
L^{n+m+1} f(x) & =\left\langle L G(x, t), L^{n+m-1} f(t)\right\rangle \\
& =\left\langle L^{n} G(x, t), L^{m} f(t)\right\rangle, \quad x \in[a, b], \\
& n, m \geq 0 .
\end{aligned}
$$

Equation (133) is a particular case of (132) for $m=0$.

Remark 27. The advantage of (133) is that, given (2) and fixing the value $k$ we want to apply to $L^{k}$, it allows testing easily different functions $f, g$ (as many as we want) in Theorems 7 and 18-23 in a simple way, just leaving the complication of the method to the calculation of $L^{k-1} G(x, t)$.

\section{Some Examples}

Throughout this section we will introduce examples where Theorems 7 and 12 and Corollary 20 will be used to determine upper and lower bounds for the distance between extremes $a$ and $b$ of a solution of (2) for different functions $q(x)$ and boundary conditions (3). For the sake of simplicity, the analysis will fix the value of the starting point $a$ (in all cases zero) and will search for upper and lower bounds of the adjacent right extreme $b$. The boundary conditions addressed will be disfocality, in the first three examples, and disconjugacy, in the two last ones, and the examples used to illustrate the disfocal case will be the same as those that were introduced in [1], in order to compare the bounds obtained via Theorems 7 and 12 with those coming from Corollary 20, which was already obtained in [1] for the disfocal case. A comparison between these bounds and the bounds obtained via other existing methods, like Brown and Hinton's one (see [24]) for lower bounds, will also be done.

In all examples the calculation of $L^{k}$ will be done numerically and the number of iterations will be restricted case by case.

Example 1. Let us consider the following boundary value problem

$$
y^{\prime \prime}+A e^{x}=0, \quad x \geq 0, \quad y(0)=0, \quad y^{\prime}(b)=0,
$$

for different values of the constant $A$.

The application of Theorem 7 to the function $f(x)=x$, together with Theorem 12 and Corollary 20 (already applied to this problem in [1] with $f(x)=(x / b)$ and $f(x)$ equal to the function $\Phi(x)$ of $[1$, Equation (47)]) gives Table 1 .

As can be seen from Table 1, Theorem 7 (i.e., $\left\|L^{k}\{x\}\right\|_{2}^{2}<$ $\|x\|_{2}^{2}$ ) provides better upper bounds of $b$ than Corollary 20 (that is, $L^{k}\{x / b\}(b)<1$ ) with a lesser number of iterations for all values of the constant $A$. On the other hand, Theorem 12
TABLE 1: Comparison of bounds for $b$ in Example 1.

\begin{tabular}{lll}
\hline Value of $A$ & Used formula & Bound \\
\hline & $L^{10}\left\{\frac{x}{b}\right\}(b)<1$ & $b<1.072$ \\
& $L^{15}\{\Phi\}(b)>1$ & $b>1.0635$ \\
& $L^{17}\{\Phi\}(b)>1$ & $b>1.0637$ \\
& Brown and Hinton & $b>1.0635$ \\
& $\left\|L^{2}\{x\}\right\|_{2}^{2}<\|x\|_{2}^{2}$ & $b<1.067$ \\
& Theorem 12 with $k=25$ & $b>1.0627$ \\
\hline \multirow{4}{*}{$A=3$} & $L^{6}\left\{\frac{x}{b}\right\}(b)<1$ & $b<0.712$ \\
& $L^{9}\{\Phi\}(b)>1$ & $b>0.7020$ \\
& $L^{12}\{\Phi\}(b)>1$ & $b>0.7025$ \\
& Brown and Hinton & $b>0.7020$ \\
& $\left\|L^{2}\{x\}\right\|_{2}^{2}<\|x\|_{2}^{2}$ & $b<0.705$ \\
& Theorem 12 with $k=24$ & $b>0.7023$ \\
\hline & $L^{6}\left\{\frac{x}{b}\right\}(b)<1$ & $b<0.580$ \\
& $L^{8}\{\Phi\}(b)>1$ & $b>0.5707$ \\
& $L^{12}\{\Phi\}(b)>1$ & $b>0.5712$ \\
& Brown and Hinton & $b>0.5707$ \\
& $\left\|L^{2}\{x\}\right\|_{2}^{2}<\|x\|_{2}^{2}$ & $b<0.573$ \\
& Theorem 12 with $k=17$ & $b>0.5707$ \\
\hline
\end{tabular}

yields lower bounds of $b$ which are either worse than Corollary 20 and Brown and Hinton's method [24] or, when equal, require a higher number of iterations than [1]. And as was mentioned in [1], it is worth remarking the good accuracy that Brown and Hinton's method gives for lower bounds with relatively low calculation effort.

Example 2. Let us consider the following boundary value problem:

$$
y^{\prime \prime}+B x y=0, \quad x \geq 0, \quad y(0)=0, \quad y^{\prime}(b)=0,
$$

for different values of the constant $B$.

The application of Theorem 7 to the function $f(x)=x$, together with Theorem 12 and Corollary 20 (already applied to this problem in [1]), gives Table 2 , where one can observe results and trends similar to those commented in the previous example.

Example 3. Let us consider the following boundary value problem:

$$
y^{\prime \prime}+C e^{-x} y=0, \quad x \geq 0, \quad y(0)=0, \quad y^{\prime}(b)=0,
$$

for different values of the constant $C$.

The application of Theorem 7 to the function $f(x)=x$, together with Theorem 12 and Corollary 20 (already applied to this problem in [1]), gives Table 3.

In this third example one can observe that Theorem 12 finally gets to yield greater (i.e., better) lower bounds of $b$ than those provided by Corollary 20 and Brown and Hinton's method [24] with a lesser number of iterations $k$. 
TABLE 2: Comparison of bounds for $b$ in Example 2.

\begin{tabular}{lll}
\hline Value of $B$ & Used formula & Bound \\
\hline \multirow{4}{*}{$B=1$} & $L^{5}\left\{\frac{x}{b}\right\}(b)<1$ & $b<1.530$ \\
& $L^{22}\{\Phi\}(b)>1$ & $b>1.513$ \\
& Brown and Hinton & $b>1.513$ \\
& $\left\|L^{2}\{x\}\right\|_{2}^{2}<\|x\|_{2}^{2}$ & $b<1.516$ \\
& Theorem 12 with $k=24$ & $b>1.5106$ \\
\hline \multirow{4}{*}{$3=3$} & $L^{5}\left\{\frac{x}{b}\right\}(b)<1$ & $b<1.062$ \\
& $L^{23}\{\Phi\}(b)>1$ & $b>1.049$ \\
& Brown and Hinton & $b>1.049$ \\
& $\left\|L^{3}\{x\}\right\|_{2}^{2}<\|x\|_{2}^{2}$ & $b<1.0508$ \\
& Theorem 12 with $k=23$ & $b>1.0473$ \\
\hline \multirow{4}{*}{$=5$} & $L^{5}\left\{\frac{x}{b}\right\}(b)<1$ & $b<0.894$ \\
& $L^{23}\{\Phi\}(b)>1$ & $b>0.884$ \\
& Brown and Hinton & $b>0.884$ \\
& $\left\|L^{6}\{x\}\right\|_{2}^{2}<\|x\|_{2}^{2}$ & $b<0.886$ \\
& Theorem 12 with $k=25$ & $b>0.8835$ \\
\hline
\end{tabular}

TABLE 3: Comparison of bounds for $b$ in Example 3.

\begin{tabular}{lll}
\hline Value of $C$ & Used formula & Bound \\
\hline & $L^{7}\left\{\frac{x}{b}\right\}(b)<1$ & $b<1.007$ \\
& $L^{10}\{\Phi\}(b)>1$ & $b>0.969$ \\
& $L^{16}\{\Phi\}(b)>1$ & $b>0.972$ \\
& Brown and Hinton & $b>0.969$ \\
& $\left\|L^{3}\{x\}\right\|_{2}^{2}<\|x\|_{2}^{2}$ & $b<0.98$ \\
& Theorem 12 with $k=7$ & $b>0.970$ \\
& Theorem 12 with $k=19$ & $b>0.974$ \\
\hline \multirow{4}{*}{10} & $L^{5}\left\{\frac{x}{b}\right\}(b)<1$ & $b<0.633$ \\
& $L^{8}\{\Phi\}(b)>1$ & $b>0.609$ \\
& $L^{15}\{\Phi\}(b)>1$ & $b>0.611$ \\
& Brown and Hinton & $b>0.609$ \\
& $\left\|L^{2}\{x\}\right\|_{2}^{2}<\|x\|_{2}^{2}$ & $b<0.6161$ \\
& Theorem 12 with $k=4$ & $b>0.609$ \\
& Theorem 12 with $k=24$ & $b>0.6119$ \\
\hline & $L^{5}\left\{\frac{x}{b}\right\}(b)<1$ & $b<0.415$ \\
& $L^{5}\{\Phi\}(b)>1$ & $b>0.401$ \\
& $L^{14}\{\Phi\}(b)>1$ & $b>0.403$ \\
& Brown and Hinton & $b>0.401$ \\
& $\left\|L^{2}\{x\}\right\|_{2}^{2}<\|x\|_{2}^{2}$ & $b<0.405$ \\
& Theorem 12 with $k=11$ & $b>0.403$ \\
& Theorem 12 with $k=21$ & $b .4033$ \\
\hline & &
\end{tabular}

Example 4. Let us consider the following boundary value problem:

$$
y^{\prime \prime}+A e^{x}=0, \quad x \geq 0, \quad y(0)=0, \quad y(b)=0,
$$

for different values of the constant $A$.
TABLE 4: Comparison of bounds for $b$ in Example 4.

\begin{tabular}{lll}
\hline Value of $A$ & Used formula & Bound \\
\hline \multirow{4}{*}{$A=3$} & $\int_{a}^{b} q(t) G(t, t) L^{11}\{1\}(t) d t>1$ & $b>1.266$ \\
& Theorem 12 with $k=11$ & $b>1.279$ \\
& $\left\|L^{5}\{v\}\right\|_{2}^{2}<\|v\|_{2}^{2}$ & $b<1.287$ \\
& $L^{10}\{v\}\left(\frac{a+b}{2}\right)<1$ & $b<1.298$ \\
\hline \multirow{4}{*}{$A=5$} & $\int_{a}^{b} q(t) G(t, t) L^{11}\{1\}(t) d t>1$ & $b>1.044$ \\
& $T^{T} L^{5}\{v\}\left\|_{2}^{2}<\right\| v \|_{2}^{2}$ & $b>1.056$ \\
& $L^{10}\{v\}\left(\frac{a+b}{2}\right)<1$ & $b<1.062$ \\
&
\end{tabular}

The application of Theorem 7 and Corollary 20-(118)to the function $f(x)$ defined by

$$
f(x)=v(x)= \begin{cases}\frac{2 x}{b} & x \in\left[0, \frac{b}{2}\right], \\ \frac{2(b-x)}{b} & x \in\left[\frac{b}{2}, b\right] ;\end{cases}
$$

together with Theorem 12 and Corollary 20-(117) - gives Table 4.

In this example the methods based on comparison of norms yield better results than those based on comparison of values of $L^{k}$. The reason for this may be that the boundary conditions of this case do not allow knowing in advance the value of the maximum of $L^{k}$ to be used in the comparison (note that in the three previous examples the maximum was always placed at $b)$, causing the selection of $((a+b) / 2)$ as comparison value in the case of Corollary 20-(118) - and the use of $G(t, t)$ in the integral in the case of Corollary $20-$ (117) - both of which require further iterations to surpass the threshold value 1 .

Example 5. Let us consider the following boundary value problem

$$
y^{\prime \prime}+B x y=0, \quad x \geq 0, \quad y(0)=0, \quad y(b)=0,
$$

for different values of the constant $B$.

The application of Theorem 7 and Corollary 20-(118)to the function $v(x)$ defined by (140) together with Theorem 12 and Corollary 20-(117) - gives Table 5, where one can observe results and trends similar to those commented in the previous example.

\section{Conclusions}

Throughout this paper two different types of methods have been provided to assess whether there are nontrivial solutions of (2) satisfying the boundary conditions (3) at extremes $a^{\prime}$, $b^{\prime}$ inner, equal, or outer to other fixed extremes $a, b$. Both rely on an iterative application of the operator $L$ defined in 
TABLE 5: Comparison of bounds for $b$ in Example 5.

\begin{tabular}{|c|c|c|}
\hline Value of $B$ & Used formula & Bound \\
\hline \multirow{4}{*}{$B=3$} & $\int_{a}^{b} q(t) G(t, t) L^{11}\{1\}(t) d t>1$ & $b>1.825$ \\
\hline & Theorem 12 with $k=12$ & $b>1.838$ \\
\hline & $\left\|L^{7}\{v\}\right\|_{2}^{2}<\|v\|_{2}^{2}$ & $b<1.85$ \\
\hline & $L^{10}\{v\}\left(\frac{a+b}{2}\right)<1$ & $b<1.866$ \\
\hline \multirow{4}{*}{$B=5$} & $\int_{a}^{b} q(t) G(t, t) L^{11}\{1\}(t) d t>1$ & $b>1.539$ \\
\hline & Theorem 12 with $k=12$ & $b>1.55$ \\
\hline & $\left\|L^{9}\{v\}\right\|_{2}^{2}<\|v\|_{2}^{2}$ & $b<1.56$ \\
\hline & $L^{10}\{v\}\left(\frac{a+b}{2}\right)<1$ & $b<1.574$ \\
\hline
\end{tabular}

(4) and allow obtaining sequences of extremes $a_{k}, b_{k}$ that converge to the exact values $a, b$ for which $y(x)$ satisfies (3). The second characteristic is by far the most relevant one when one compares them with other methods present in the literature, since these latter only provide bounds (in fact very often only lower bounds or Lyapunov inequalities since the number of methods dealing with upper bounds is quite low) which cannot be improved following the same approach.

In addition it is worth remarking that the set of methods presented over almost all possible boundary conditions (3), despite the fact that some of them (namely, the method based on Theorem 12 and those based on Theorems 18 and 19) only apply under a limited subset of boundary conditions (3). The reason for this is, on the one hand, that the only conditions common for all these methods are those of Lemma 3 (which indeed are relatively easy to satisfy) and, on the other hand, that Theorem 7 does not require any additional conditions at all and Theorem 12 requires additional conditions on (3) which complement those required by Theorems 18 and 19 .

As for what method is better in what case, it is difficult to decide, but the examples show that in the quest for outer bounds the method based on Theorem 7 converges normally much faster than the one based on Theorem 18. In the search of inner bounds, as indicated before, Theorems 12 and 19 cannot be applied simultaneously to most of the boundary conditions (3), but in the cases where that is possible, the speed of convergence depends on the concrete boundary conditions and the concrete function $q(x)$. In general it seems that boundary conditions aiming for a maximum in $L^{k}$ different from $a$ or $b$ tend to favour the method based on Theorem 12 versus the method based on Theorem 19, the opposite occurring (with exceptions, as Example 3 shows) when the boundary conditions force maxima of $L^{k}$ at either $a$ or $b$.

As for the main drawbacks, we can comment on two. On the one hand, the fact that the speed of convergence of the method depends vastly on the selected starting function $f$ and can be low in some cases, many iterations being necessary to approximate the values of the extremes $a$ and $b$ that satisfy (3). On the other hand, as they have been presented, the methods cannot deal with functions $q(x)$ which are zero or negative in a subset of positive measure. However it can be shown, as was done in [1], that these methods can be extended to the case $q(x)$ zero or negative in a way similar to most of the criteria published so far for the same problem.

All in all and taking the mentioned constraints into consideration, we the authors believe that the advantages of the methods presented in this paper surpass their drawbacks largely and that they can become a very powerful tool to assess problems of conjugacy/disconjugacy, nondisfocality/disfocality, and so forth, of (2), whether directly or by means of other methods based on them which in turn improve them.

\section{Conflict of Interests}

The authors declare that there is no conflict of interests regarding the publication of this paper.

\section{Acknowledgment}

This work has been supported by the Spanish Ministry of Science and Innovation Project DPI2010-C02-01.

\section{References}

[1] P. Almenar and L. Jódar, "Convergent disfocality and nondisfocality criteria for second order linear differential equations," Abstract and Applied Analysis, vol. 2013, Article ID 987976, 11 pages, 2013.

[2] Z. Nehari, "On the zeros of solutions of second-order linear differential equations," American Journal of Mathematics, vol. 76, no. 3, pp. 689-697, 1954.

[3] P. Beesack, "On the Green's function of an N-point boundary value problem," Pacific Journal of Mathematics, vol. 12, no. 3, pp. 801-812, 1962.

[4] D. B. Hinton, "Disconjugate properties of a system of differential equations," Journal of Differential Equations, vol. 2, no. 4, pp. 420-437, 1966.

[5] A. J. Levin, "Some problems concerning the oscillation of solutions of linear differential equations," Soviet Mathematics Doklady, vol. 4, pp. 121-124, 1963.

[6] A. J. Levin, "Distribution of zeros of solutions of linear differential equations," Soviet Mathematics Doklady, vol. 5, pp. 818-822, 1964.

[7] W. Reid, "A matrix Liapunov inequality," Journal of Mathematical Analysis and Applications, vol. 32, no. 2, pp. 424-434, 1970.

[8] W. Reid, "A generalized Liapunov inequality," Journal of Differential Equations, vol. 13, no. 1, pp. 182-196, 1973.

[9] W. Reid, "Interrelations between a trace formula and Liapunov type inequalities," Journal of Differential Equations, vol. 23, no. 3, pp. 448-458, 1977.

[10] T. M. Rassias, Survey on Classical Inequalities, vol. 517 of Mathematics and Its Applications, Kluwer Academic, Dodrecht, The Netherlands, 2000.

[11] B. J. Harris, "On an inequality of Lyapunov for disfocality," Journal of Mathematical Analysis and Applications, vol. 146, no. 2, pp. 495-500, 1990.

[12] O. Došlý, "Conjugacy criteria for second order differential equations," The Rocky Mountain Journal of Mathematics, vol. 23, no. 3, pp. 849-861, 1993. 
[13] P. Almenar and L. Jódar, "An upper bound for the distance between a zero and a critical point of a solution of a second order linear differential equation," Computers \& Mathematics with Applications, vol. 63, no. 1, pp. 310-317, 2012.

[14] P. Almenar and L. Jódar, "The distribution of zeroes and critical points of solutions of a second order half-linear differential equation," Abstract and Applied Analysis, vol. 2013, Article ID 147192, 6 pages, 2013.

[15] S. W. Hawking and R. Penrose, "The singularities of gravitational collapse and cosmology," Proceedings of the Royal Society of London Series A: Mathematical, Physical \& Engineering Sciences, vol. 314, no. 1519, pp. 529-548, 1970.

[16] F. J. Tipler, "General relativity and conjugate ordinary differential equations," Journal of Differential Equations, vol. 30, no. 2, pp. 165-174, 1978.

[17] O. Došlý and J. Komenda, "Conjugacy criteria and principal solutions of self-adjoint differential equations," Archivum Mathematicum, vol. 31, no. 3, pp. 217-238, 1995.

[18] O. Došlý, "The multiplicity criteria for zero points of second order differential equations," Mathematica Slovaca, vol. 42, no. 2, pp. 181-193, 1992.

[19] E. Müller-Pfeiffer, "Existence of conjugate points for second and fourth order differential equations," Proceedings of the Royal Society of Edinburgh A: Mathematics, vol. 89, no. 3-4, pp. 281291, 1981.

[20] E. Müller-Pfeiffer, "Nodal domains for one- and twodimensional elliptic differential equations," Zeitschrift für Analysis und Ihre Anwendungen, vol. 7, no. 2, pp. 135-139, 1988.

[21] V. Hutson, J. S. Pym, and M. J. Cloud, Applications of Functional Analysis and Operator Theory, vol. 200 of Mathematics in Science and Engineering, Elsevier, Amsterdam, The Netherlands, 2nd edition, 2005.

[22] H. Sagan, Boundary and Eigenvalue Problems in Mathematical Physics, Dover, New York, NY, USA, 1989.

[23] R. Moore, "The behavior of solutions of a linear differential equation of second order," Pacific Journal of Mathematics, vol. 5, no. 1, pp. 125-145, 1955.

[24] R. C. Brown and D. B. Hinton, "Opial's inequality and oscillation of 2nd order equations," Proceedings of the American Mathematical Society, vol. 125, no. 4, pp. 1123-1129, 1997. 


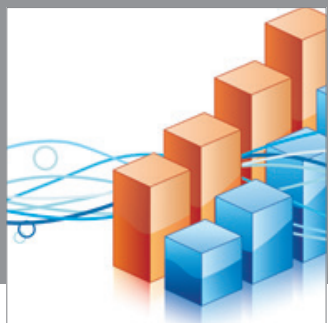

Advances in

Operations Research

mansans

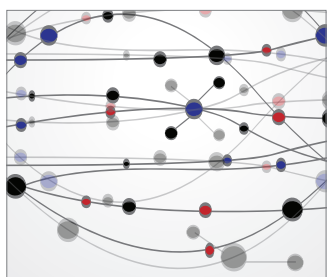

The Scientific World Journal
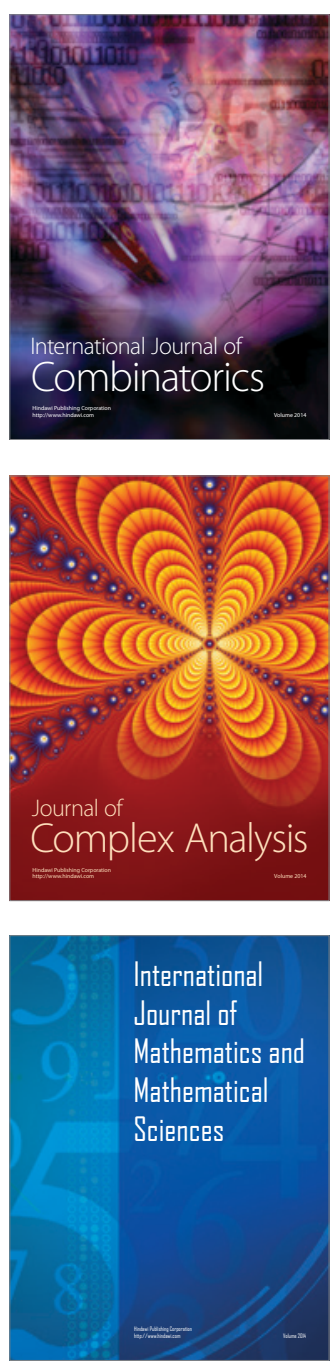
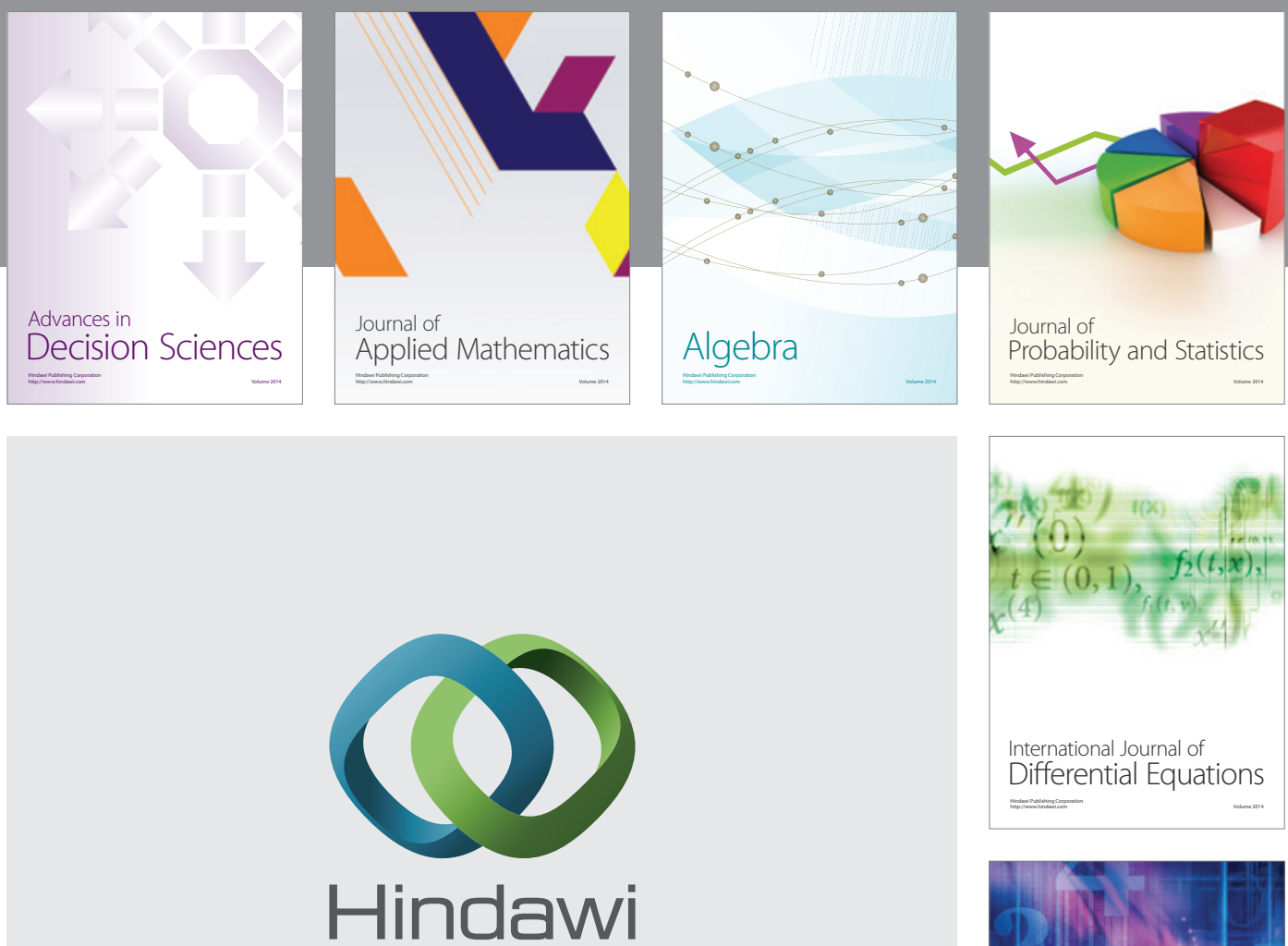

Submit your manuscripts at http://www.hindawi.com
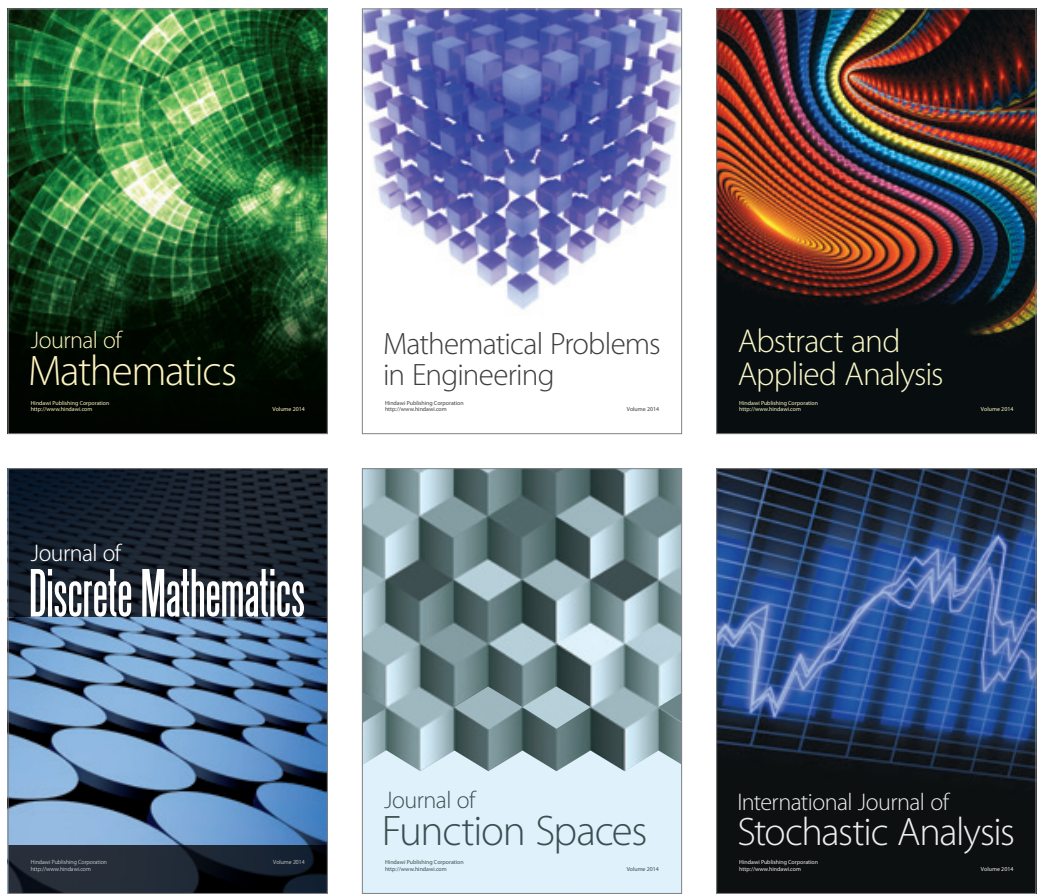

Journal of

Function Spaces

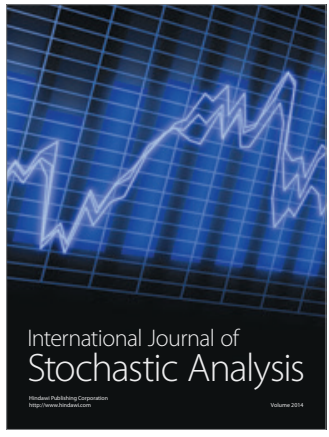

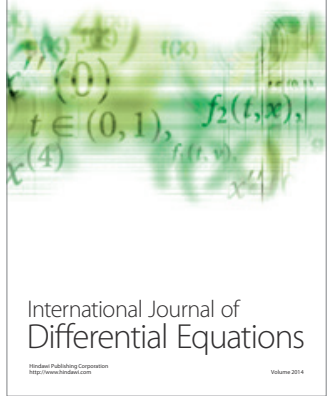
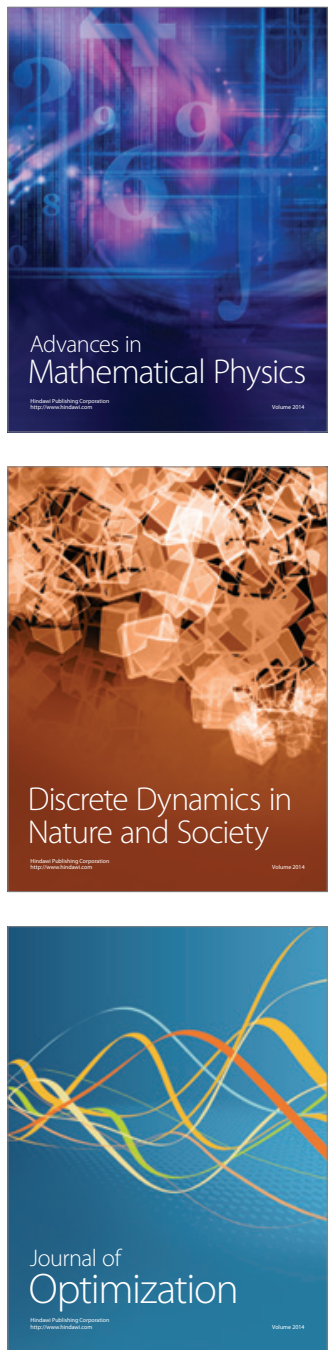